\title{
Modeling thin curved ferromagnetic films
}

\author{
Hamdi Zorgati ${ }^{1}$
}

May 24, 2005

\begin{abstract}
The behavior of a thin film made of a ferromagnetic material in the absence of an external magnetic field, is described by an energy depending on the magnetization of the film verifying the saturation constraint. The free energy consists of an induced magnetostatic energy and an energy term with density including the exchange energy and the anisotropic energy. We study the behavior of this energy when the thickness of the curved film goes to zero. We show that the minimizers of the free energy converge to the minimizers of a local energy depending on a two-dimensional magnetization by $\Gamma$-convergence arguments.
\end{abstract}

\section{Introduction}

The theory of micromagnetism developed by L.D. Landau, E.M. Lifschitz [9] and W.F. Brown [3], describes the magnetic behavior of ferromagnetic bodies. According to this theory, the equilibrium state of a ferromagnetic body is described by its magnetization $m$, wich is a vector field defined on $\mathbb{R}^{3}$, vanishing outside the body. The magnetization represents the volume density of macroscopic magnetic moment. This means that $m$ induces a magnetic field in all the space. When a ferromagnetic body occupying a domain $\Omega$ is submitted to an external magnetic field $H_{e}$, the observed states are the minimizers of an energy $E_{e}$ depending on the magnetization $m$ :

$$
E_{e}(m)=\int_{\Omega}\left\{\alpha|\nabla m|^{2}+Q \varphi(m)+H_{e} \cdot m\right\}+\int_{\mathbb{R}^{3}} \frac{1}{2}|H|^{2} .
$$

${ }^{1}$ CEREMADE, CNRS UMR 7534, Université Paris-Dauphine, Place du Maréchal de Lattre de Tassigny, 75775, Paris, France \& Laboratoire Jacques-Louis Lions, Université Pierre et Marie Curie, 75252 Paris Cedex 05, France, email: zorgati@ann.jussieu.fr 
The first term in the free energy represents the exchange energy. It penalizes the spatial variations of the magnetization $m$ in order to model the tendency of a specimen to exhibit regions of uniform magnetization (magnetic domains) separated by very thin transition layers (domain walls). The constant $\alpha$ is a non-negative constant. The second term is the anisotropic energy term. It models the existence of preferred directions of the magnetization (easy axes), along which $\varphi$, which is supposed to be a nonnegative, even function exhibiting cristallographic symmetry, vanishes. In general, $\varphi$ is supposed to be a polynomial function. The coefficient $Q$ controls the relative importance of the anisotropic energy compared to the magnetostatic energy. The magnetostatic energy, that is the fourth term of the free energy, is induced by the magnetization $m$, where $H=-\nabla u$ is the magnetic field induced by $m$ in $\mathbb{R}^{3}$, and $u$ is a scalar potential verifying the magnetostatic equation

$$
\operatorname{div}(-\nabla u+m)=0 \text { in } \mathbb{R}^{3} .
$$

The third term composing the free energy is the external energy or the Zeeman energy. It is due to the external magnetic field in which the ferromagnetic body is placed. More details can be found in A. De Simone [6]. At constant temperature the magnetization $m$ verifies the saturation condition

$$
|m|=M \text { in } \Omega,
$$

where $M$ is a nonnegative constant that we suppose equal to 1 .

The study of ferromagnetic thin films is of great interest, because this type of material is found in several fields in industry, such as audio and video tapes containing ferromagnetic ribbons used to provide high density in audio or video recording.

The first mathematical works on ferromagnetic films concerned wide films with constant thickness, see A. De Simone [6], B. Dacorogna and I. Fonseca [4]. Then, G. Gioia and R.D. James [8] studied the behavior of a thin ferromagnetic film, with no external magnetic field, when its thickness goes to zero. Gioia and James considered a thin film of thickness $h$ occupying the domain $\Omega_{h}$. The film has an energy per unit volume with no external energy term. They show that the magnetization minimizing the free energy of the thin film converges, when the thickness goes to zero, to a magnetization minimizing a limit energy. This limit energy is local, which means that there is no magnetostatic equation in the limit model. Gioia and James also show that the limit magnetization is independent of the direction normal to the film, wich means that the limit model is two-dimentional. In [2], R. Alicandro and C. Leone extended the study of Gioia and James considering a more general density $W$, depending on the magnetization and its gradient, verifying certain growth hypotheses and including the exchange and anisotropic energies. Alicandro and Leone used the concept of tangential 
quasiconvexity introduced by B. Dacorogna, I. Fonseca, J. Maly and K. Trivisa [5] in order to compute the relaxation of a class of energy functionals where the admissible fields are constrained to remain in a $C^{1}, q$-dimensional manifold of $\mathbb{R}^{d}$.

In this paper, we follow [2] and we use $\Gamma$-convergence arguments to study the behavior of the energy of a curved ferromagnetic thin film and its minimizers when the thickness of the film goes to zero. After setting the problem and rescaling the energy, we study the magnetostatic energy term by examining the magnetostatic potential solution of the equation (1.1). We get the behavior of the magnetostatic energy setting a new minimization problem. Then, we recall the notion of tangential quasiconvexity before computing the $\Gamma$-limit of the sequence of energies that gives the behavior of almost minimizing sequences. Next, we rewrite the limit model on the curved surface following [11]. Finally, we apply our results to the Gioia and James model and to the model with external magnetic field. These results were announced in [14]

\section{Notation and geometrical preliminaries}

Throughout this article, we assume the summation convention unless otherwise specified. Greek indices take their values in the set $\{1,2\}$ and Latin indices in the set $\{1,2,3\}$.

Let $\left(e_{1}, e_{2}, e_{3}\right)$ be the canonical orthonormal basis of the Euclidean space $\mathbb{R}^{3}$. We denote by $|v|$ the norm of a vector $v$ in $\mathbb{R}^{3}$, by $u \cdot v$ the scalar product of two vectors in $\mathbb{R}^{3}$, by $u \wedge v$ their vector product and by $u \otimes v$ their tensor product. Let $\mathbb{M}_{33}$ be the space of $3 \times 3$ real matrices endowed with the usual norm $|F|=\sqrt{\operatorname{tr}\left(F^{T} F\right)}$. This is a matrix norm in the sense that $|A B| \leq|A||B|$. We denote by $A=\left(a_{1}\left|a_{2}\right| a_{3}\right)$ the matrix in $\mathbb{M}_{33}$ whose $i$ th column is $a_{i}$. We consider a thin curved film of thickness $h>0$ occupying at rest an open domain $\widetilde{\Omega}_{h}$. This reference configuration of the film is described as follows. We are thus given a surface $\widetilde{S}$, called the midsurface of the film. This surface is a bounded two-dimensional $C^{1}$-submanifold of $\mathbb{R}^{3}$ and we assume for simplicity that it admits an atlas consisting of one chart. Let $\psi$ be this chart, i.e. a $C^{1}$-diffeomorphism from a bounded open subset $\omega$ of $\mathbb{R}^{2}$ onto $\widetilde{S}$.

Let $a_{\alpha}(x)=\psi_{, \alpha}(x)$ be the vectors of the covariant basis of the tangent plane $T_{\psi(x)} \widetilde{S}$ associated with the chart $\psi$, where $\psi_{, \alpha}$ denotes the partial derivative of $\psi$ with respect to $x_{\alpha}$. We assume that there exists $\delta>0$ such that $\left|a_{1}(x) \wedge a_{2}(x)\right| \geq \delta$ on $\bar{\omega}$ and we define the unit normal vector $a_{3}(x)=\frac{a_{1}(x) \wedge a_{2}(x)}{\left|a_{1}(x) \wedge a_{2}(x)\right|}$, which belongs to $C^{1}\left(\bar{\omega}, \mathbb{R}^{3}\right)$. The vectors $a_{1}(x), a_{2}(x)$ and $a_{3}(x)$ constitute the covariant basis at the point $x$. We define the contravariant basis by the relations $a^{i}(x) \cdot a_{j}(x)=\delta_{j}^{i}$, so that $a^{\alpha}(x) \in T_{\psi(x)} \widetilde{S}$ and $a^{3}(x)=a_{3}(x)$. 
Next, we define a mapping $\Psi: \omega \times \mathbb{R} \rightarrow \mathbb{R}^{3}$ by

$$
\Psi\left(x_{1}, x_{2}, x_{3}\right)=\psi\left(x_{1}, x_{2}\right)+x_{3} a_{3}\left(x_{1}, x_{2}\right) .
$$

It is well known that there exists $h^{*}>0$ such that for all $0<h<h^{*}$, the restriction of $\Psi$ to $\Omega_{h}=\omega \times$ ] $-h / 2, h / 2\left[\right.$ is a $C^{1}$-diffeomorphism on its image by the tubular neighborhood theorem. For such values of $h$, we set $\widetilde{\Omega}_{h}=\Psi\left(\Omega_{h}\right)$. Alternatively, we can write

$$
\widetilde{\Omega}_{h}=\left\{\widetilde{x} \in \mathbb{R}^{3}, \exists \widetilde{\pi}(\widetilde{x}) \in \widetilde{S}, \widetilde{x}=\widetilde{\pi}(\widetilde{x})+\eta a_{3}\left(\psi^{-1}(\widetilde{\pi}(\widetilde{x}))\right) \text { with } \frac{-h}{2}<\eta<\frac{h}{2}\right\},
$$

where $\widetilde{\pi}$ denotes the orthogonal projection from $\widetilde{\Omega}_{h}$ onto $\widetilde{S}$, which is well defined and of class $C^{1}$ for $h<h^{*}$. Equivalently, every $\widetilde{x} \in \widetilde{\Omega}_{h}$ can be written as

$$
\tilde{x}=\tilde{\pi}(\tilde{x})+\left[(\widetilde{x}-\tilde{\pi}(\widetilde{x})) \cdot a_{3}\left(\psi^{-1}(\tilde{\pi}(\widetilde{x}))\right)\right] \cdot a_{3}\left(\psi^{-1}(\tilde{\pi}(\widetilde{x}))\right) .
$$

Thus, we have a curvilinear coordinate system in $\widetilde{\Omega}_{h}$ naturally associated with the chart $\psi$ by

$$
\left(x_{1}, x_{2}\right)=\psi^{-1}(\widetilde{\pi}(\widetilde{x})) \text { and } x_{3}=(\widetilde{x}-\tilde{\pi}(\widetilde{x})) \cdot a_{3}\left(\psi^{-1}(\tilde{\pi}(\widetilde{x}))\right) .
$$

Since the scalar potential $\widetilde{u}_{h}$ is defined on $\mathbb{R}^{3}$ we suppose that the middle surface $\widetilde{\Omega}_{h}$ is the image of an open domain $\omega \subset \mathbb{R}^{2}$ through a $C^{1}$-diffeomorphism $\bar{\psi}$ : $\mathbb{R}^{2} \rightarrow \mathbb{R}^{3}$ extending $\psi$ to $\mathbb{R}^{2}$. More generally, we suppose that $\Psi$ is the restriction to $\mathbb{R}^{2} \times\left(-\frac{h}{2}, \frac{h}{2}\right)$ of a $C^{1}$-diffeomorphism $\bar{\Psi}$ of $\mathbb{R}^{3}$. We can also suppose that $\Psi$ is equal to the identity outside a compact set containing $\Omega_{h}$. In what follows, we will keep the notation $\Psi$ to mean $\bar{\Psi}$.

For all $x \in \bar{\omega}$, we let $A(x)=\left(a_{1}(x)\left|a_{2}(x)\right| a_{3}(x)\right)$. We note that $A(x)$ is an invertible matrix on $\bar{\omega}$, and that its inverse is given by $A(x)^{-1}=\left(a^{1}(x)\left|a^{2}(x)\right| a^{3}(x)\right)^{T}$. We also note that $\operatorname{det} A(x)=\left|\operatorname{cof} A(x) \cdot e_{3}\right|=\left|a_{1}(x) \wedge a_{2}(x)\right| \geq \delta>0$ on $\bar{\omega}$. We clearly have

$$
\nabla \Psi\left(x_{1}, x_{2}, x_{3}\right)=A\left(x_{1}, x_{2}\right)+x_{3}\left(a_{3,1}\left(x_{1}, x_{2}\right)\left|a_{3,2}\left(x_{1}, x_{2}\right)\right| 0\right) .
$$

The matrix $\nabla \Psi\left(x_{1}, x_{2}, x_{3}\right)$ is thus everywhere invertible in $\bar{\Omega}_{h}$ and its determinant is strictly positive, and therefore equal to the Jacobian of the change of variables, for $h$ small enough.

In the following, $h$ denotes a generic sequence of real numbers in $] 0, h^{*}[$ that tends to zero. The next convergences are easily established.

Lemma 2.1 We have

$$
\left\{\begin{array}{l}
\nabla \Psi^{-1} \circ \Psi\left(x_{1}, x_{2}, h x_{3}\right) \rightarrow A(x)^{-1}, \\
\operatorname{det} \nabla \Psi\left(x_{1}, x_{2}, h x_{3}\right) \rightarrow \operatorname{det} A(x),
\end{array}\right.
$$

uniformly on $\bar{\Omega}_{1}$ when $h \rightarrow 0$. In particular, $\inf _{\bar{\Omega}_{1}} \operatorname{det} \nabla \Psi\left(x_{1}, x_{2}, h x_{3}\right) \geq \delta / 2>0$ for $h$ small enough. 


\section{The three-dimensional and rescaled problems}

The behavior of a curved ferromagnetic thin film of thickness $h$ occupying the domain $\widetilde{\Omega}_{h}$ is described by a micromagnetic energy $\widetilde{e}_{h}$ depending on its magnetization $\widetilde{m}_{h}: \mathbb{R}^{3} \rightarrow \mathbb{R}^{3}$. The vector field $\widetilde{m}_{h}$ vanishes outside the film. We refer to [6] and the references therein for more information about ferromagnetic materials. The magnetization of the film minimizes an energy $\widetilde{e}_{h}$ of the form

$$
\widetilde{e}_{h}\left(\widetilde{m}_{h}\right)=\frac{1}{h} \int_{\widetilde{\Omega}_{h}}\left[W\left(\widetilde{m}_{h}, \nabla \widetilde{m}_{h}\right)+\frac{1}{2} \nabla \widetilde{u}_{h} \cdot \widetilde{m}_{h}\right] d \widetilde{x},
$$

under the saturation constraint

$$
\left|\widetilde{m}_{h}\right|=1 \text { in } \widetilde{\Omega}_{h}
$$

The free energy is composed of an energy term with density $W$ depending on the magnetization and its gradient, that includes the exchange and anisotropic energies. The density verifies the following growth, coercivity and locally lipschitz dependence assumptions

$$
\left\{\begin{array}{l}
\exists c>0, \exists p \in] 1,+\infty\left[, \forall y \in \mathbb{R}^{3} \text { and } \forall F \in M^{3},|W(y, F)| \leq c\left(1+|F|^{p}\right),\right. \\
\exists \gamma>0, \exists \beta \geq 0, \forall y \in \mathbb{R}^{3} \text { and } \forall F \in M^{3}, W(y, F) \geq \gamma|F|^{p}-\beta, \\
\forall y \in \mathbb{R}^{3} \text { and } \forall F, F^{\prime} \in M^{3},\left|W(y, F)-W\left(y, F^{\prime}\right)\right| \leq c\left(1+|F|^{p-1}+\left|F^{\prime}\right|^{p-1}\right)\left|F-F^{\prime}\right|,
\end{array}\right.
$$

In the case of Gioia and James we have $W(y, F)=\varphi(y)+\alpha|F|^{2}$ and $p=2$.

The second term in (3.1) depends on the magnetization $\widetilde{m}_{h}$ and a scalar potential $\widetilde{u}_{h}$ for the induced magnetic field $H_{h}=-\nabla \widetilde{u}_{h}$, solution of the magnetostatic equation

$$
\operatorname{div}\left(-\nabla \widetilde{u}_{h}+\widetilde{m}_{h}\right)=0 \text { in } \mathbb{R}^{3},
$$

which we can also write $\operatorname{div} B_{h}=0$, where $B_{h}=H_{h}+\widetilde{m}_{h}$ in $\mathbb{R}^{3}$.

The observed states of the ferromagnetic film are the solutions of the minimization problem

$$
\widetilde{e}_{h}\left(\widetilde{m}_{h}\right)=\min _{\widetilde{m} \in \widetilde{V}_{h}} \widetilde{e}_{h}(\widetilde{m}),
$$

where

$$
\begin{aligned}
& \widetilde{V}_{h}=\left\{\widetilde{m} \in L^{\infty}\left(\mathbb{R}^{3} ; \mathbb{R}^{3}\right), \text { such that } \widetilde{m}_{\mid \widetilde{\Omega}_{h}} \in W^{1, p}\left(\widetilde{\Omega}_{h} ; \mathbb{R}^{3}\right),\right. \\
& \left.|\widetilde{m}|=1 \text {, a.e. in } \widetilde{\Omega}_{h} \text { and } \widetilde{m}=0 \text {, a.e. in } \widetilde{\Omega}_{h}^{c}\right\},
\end{aligned}
$$

with $p>1$. In this article we do not make any convexity assumption on $W$, consequently problem (3.3) may well not possess any solutions. Thus, we consider 
a diagonal minimizing sequence $\widetilde{m}_{h}$ for the sequence of energies $\widetilde{e}_{h}$ over the sets $\widetilde{V}_{h}$. More specifically, we assume that

$$
\widetilde{m}_{h} \in \widetilde{V}_{h} \text { and } \widetilde{e}_{h}\left(\widetilde{m}_{h}\right)=\inf _{\widetilde{m} \in \widetilde{V}_{h}} \widetilde{e}_{h}(\widetilde{m})+h \varepsilon(h),
$$

where $\varepsilon$ is a positive function such that $\varepsilon(h) \rightarrow 0$ when $h \rightarrow 0$.

Using $\Gamma$-convergence arguments, we study the behavior of the energy $\widetilde{e}_{h}$ and its almost minimizers in the above sense when the thickness of the curved film goes to zero. We begin by flattening and rescaling the minimizing problem in order to work on a fixed cylindrical domain.

In order to flatten the domain, we define $m_{h}: \mathbb{R}^{3} \rightarrow \mathbb{R}^{3}$ and $u_{h}: \mathbb{R}^{3} \rightarrow \mathbb{R}$ by setting for all $x \in \mathbb{R}^{3}, m_{h}(x)=\widetilde{m}_{h}(\Psi(x))$ and $u_{h}(x)=\widetilde{u}_{h}(\Psi(x))$. This implies that $\nabla \widetilde{m}_{h}(\widetilde{x})=\nabla m_{h}(x)\left(\nabla \Psi^{-1}(\Psi(x))\right)$ and $\nabla \widetilde{u}_{h}(\widetilde{x})=\left(\nabla \Psi^{-1}(\Psi(x))\right)^{T} \nabla u_{h}(x)$ with $\widetilde{x}=$ $\Psi(x)$. We thus set $e^{h}\left(m_{h}\right)=\widetilde{e}_{h}\left(\widetilde{m}_{h}\right)$ and we obtain

$e^{h}\left(m_{h}\right)=\frac{1}{h} \int_{\Omega_{h}}\left[W\left(m_{h}, \nabla m_{h}\left(\nabla \Psi^{-1} \circ \Psi\right)\right)+\frac{1}{2}\left(\nabla \Psi^{-1} \circ \Psi\right)^{T} \nabla u_{h} \cdot m_{h}\right] \operatorname{det} \nabla \Psi d x_{h}$.

Then, we rescale the problem by setting $m(h)(x)=m_{h}\left(x_{1}, x_{2}, h x_{3}\right)$ and $u(h)(x)=$ $u_{h}\left(x_{1}, x_{2}, h x_{3}\right)$ for all $x \in \mathbb{R}^{3}$. This implies that

$$
\nabla \widetilde{m}_{h}(\widetilde{x})=\left(m(h)_{, 1}\left|m(h)_{, 2}\right| \frac{1}{h} m(h)_{, 3}\right) A_{h} \text { and } \nabla \widetilde{u}_{h}(\widetilde{x})=A_{h}^{T} \nabla_{h} u(h),
$$

with $A_{h}(x)=\nabla \Psi^{-1} \circ \Psi\left(x_{1}, x_{2}, h x_{3}\right)$ and $\nabla_{h} u(h)=\left(\begin{array}{c}u(h)_{, 1} \\ u(h)_{, 2} \\ h^{-1} u(h)_{, 3}\end{array}\right)$. These relations may also be written $\nabla \widetilde{m}_{h}=\nabla m(h) I_{h} A_{h}$ and $\nabla \widetilde{u}_{h}=A_{h}^{T} I_{h} \nabla u(h)$ with $I_{h}=\left(\begin{array}{lll}1 & 0 & 0 \\ 0 & 1 & 0 \\ 0 & 0 & \frac{1}{h}\end{array}\right)$.

We note that $A_{h}(x)=\left(A\left(x_{1}, x_{2}\right)+h x_{3}\left(a_{3,1}\left|a_{3,2}\right| 0\right)\right)^{-1}$, for all $x \in \overline{\boldsymbol{\omega}} \times[-1,1]$ (but not necessarly outside of this domain).

Setting $e(h)(m(h))=e^{h}\left(m_{h}\right)$, we obtain

$$
\begin{aligned}
e(h)(m(h))=\int_{\Omega_{1}}\left[W \left(m(h),\left(m(h)_{, 1}\left|m(h)_{, 2}\right| h^{-1}\right.\right.\right. & \left.\left.m(h)_{, 3}\right) A_{h}\right) \\
& \left.+\frac{1}{2} A_{h}^{T} \nabla_{h} u(h) \cdot m(h)\right] d_{h} d x,
\end{aligned}
$$

with $d_{h}(x)=\operatorname{det} \nabla \Psi\left(x_{1}, x_{2}, h x_{3}\right)$.

The magnetostatic equation then reads

$$
\left(\nabla\left(-A_{h}^{T}(x) I_{h} \nabla u(h)(x)+m(h)(x)\right)\right):\left(A_{h}^{T}(x) I_{h}\right)=0 \text { in } \mathbb{R}^{3} .
$$


and the saturation constraint reads

$$
|m(h)(x)|=1 \text { for all } x \in \Omega_{1} .
$$

\section{Magnetostatic energy behavior}

In this section we study the behavior of the magnetostatic energy term

$$
E_{\mathrm{mag}}(h)(m)=\int_{\Omega_{1}} \frac{1}{2} A_{h}^{T} \nabla_{h} u \cdot m d_{h} d x,
$$

when the thickness of the curved film goes to zero. Let us thus be given $m: \mathbb{R}^{3} \rightarrow$ $\mathbb{R}^{3}$ such that $m_{\mid \Omega_{1}} \in L^{\infty}\left(\Omega_{1} ; S^{2}\right)$ and $m=0$ on $\Omega_{1}^{c}$. We consider the minimization problem: Find $u(h, m) \in U$ such that

$$
I_{m}(h)(u(h, m))=\inf _{v \in U} I_{m}(h)(v),
$$

with

$$
I_{m}(h)(v)=\frac{1}{2} \int_{\mathbb{R}^{3}}\left|A_{h}^{T} \nabla_{h} v-m\right|^{2} d_{h} d x
$$

and

$$
U=\left\{v \in L_{\mathrm{loc}}^{1}\left(\mathbb{R}^{3}\right), \nabla v \in L^{2}\left(\mathbb{R}^{3} ; \mathbb{R}^{3}\right), \int_{B} v d x=0\right\},
$$

where $B$ is the unit ball of $\mathbb{R}^{3}$. The condition $\int_{B} v d x=0$ excludes the trivial translations $v \rightarrow v+c$. We endow $U$ with the scalar product

$$
(u, v)_{U, h}=\int_{\mathbb{R}^{3}} A_{h}^{T} \nabla_{h} u \cdot A_{h}^{T} \nabla_{h} v d_{h} d x .
$$

When endowed with this scalar product, $U$ is a Hilbert space (see [13]). Thus, problem (4.1) has a unique solution $u(h, m) \in U$ verifying the following EulerLagrange equation

$$
\int_{\mathbb{R}^{3}}\left(A_{h}^{T} \nabla_{h} u(h, m)-m\right) \cdot A_{h}^{T} \nabla_{h} v d_{h} d x=0, \forall v \in U,
$$

that is the weak form of (3.4). Setting $v=u(h, m)$ in (4.2), we obtain

$$
\int_{\mathbb{R}^{3}}\left|A_{h}^{T} \nabla_{h} u(h, m)\right|^{2} d_{h} d x=\int_{\mathbb{R}^{3}} A_{h}^{T} \nabla_{h} u(h, m) \cdot m d_{h} d x,
$$

where the right-hand side is equal to twice the magnetostatic energy term $E_{\operatorname{mag}}(h)$ corresponding to a given magnetization $m$. The following proposition gives the behavior of the magnetostatic energy term under convergence of the magnetization. 
Proposition 4.1 Let $\bar{m}(h)$ be a sequence of functions in $L^{\infty}\left(\mathbb{R}^{3} ; \mathbb{R}^{3}\right)$ such that $\bar{m}(h)=0$ on $\Omega_{1}^{c}$ and $|\bar{m}(h)|=1$ on $\Omega_{1}$, verifying $\bar{m}(h) \rightarrow \bar{m}(0)$ strongly in $L^{2}\left(\mathbb{R}^{3} ; \mathbb{R}^{3}\right)$. Let $\bar{u}(h, \bar{m}(h))$ be the solution of the minimizing problem (4.1) associated to $\bar{m}(h)$. Then, we have

$$
\nabla \bar{u}(h, \bar{m}(h)) \rightarrow 0 \text { in } L^{2}\left(\mathbb{R}^{3} ; \mathbb{R}^{3}\right) \text { and } \frac{1}{h} \bar{u}(h, \bar{m}(h))_{, 3} \rightarrow w \quad \text { in } L^{2}\left(\mathbb{R}^{3}\right),
$$

where $w \in L^{2}\left(\mathbb{R}^{3}\right)$ verifies

$$
\int_{\mathbb{R}^{3}}\left|w \widetilde{a}^{3}\right|^{2} d_{0} d x=\int_{\Omega_{1}}\left|a_{3} \cdot \bar{m}(0)\right|^{2} d_{0} d x,
$$

and $\widetilde{a}^{3}$ represents the third column vector of $A_{0}^{T}(x)=\nabla \Psi^{-1}\left(\Psi\left(x_{1}, x_{2}, 0\right)\right)$ which is equal to $a_{3}$ on $\Omega_{1}$. We have also,

$$
E_{\mathrm{mag}}(h)(\bar{m}(h)) \rightarrow E_{\mathrm{mag}}(0)(\bar{m}(0))=\frac{1}{2} \int_{\Omega_{1}}\left|\left(a_{3}, \bar{m}(0)\right)\right|^{2} d_{0} d x .
$$

We recall that $a_{3}$ is the third vector of the covariant basis associated to the diffeomorphism $\Psi$ on $\Omega_{1}$.

Proof Since $\bar{u}(h, \bar{m}(h))$ minimizes $I_{\bar{m}(h)}^{h}$ over $U$ and $0 \in U$, we have

$$
\begin{aligned}
I_{\bar{m}(h)}(h)(\bar{u}(h, \bar{m}(h))) \leq I_{\bar{m}(h)}(0) & =\frac{1}{2} \int_{\mathbb{R}^{3}}|\bar{m}(h)|^{2} d_{h} d x \\
& =\frac{1}{2} \operatorname{vol}\left(\widetilde{\Omega}_{h}\right) \leq c .
\end{aligned}
$$

Using the triangle inequality, we obtain that

$$
\left(\int_{\mathbb{R}^{3}}\left|A_{h}^{T} \nabla_{h} \bar{u}(h, \bar{m}(h))\right|^{2} d_{h} d x\right)^{\frac{1}{2}} \leq c+\left(\int_{\Omega_{1}} d_{h} d x\right)^{\frac{1}{2}} \leq c^{\prime} .
$$

Since

$$
\left|\nabla_{h} \bar{u}(h, \bar{m}(h))\right| \leq\left\|A_{h}^{-T}\right\|_{L^{\infty}\left(\mathbb{R}^{3} ; M^{3}\right)}\left|A_{h}^{T} \nabla_{h} \bar{u}(h, \bar{m}(h))\right|
$$

we have

$$
\|\nabla \bar{u}(h, \bar{m}(h))\|_{L^{2}\left(\mathbb{R}^{3} ; \mathbb{R}^{3}\right)} \leq c \quad \text { and } \quad \frac{1}{h}\left\|\bar{u}(h, \bar{m}(h))_{, 3}\right\|_{L^{2}\left(\mathbb{R}^{3}\right)} \leq c .
$$

Thus, for a subsequence still denoted $h$, there exists $H \in L^{2}\left(\mathbb{R}^{3} ; \mathbb{R}^{3}\right)$ and $w \in$ $L^{2}\left(\mathbb{R}^{3}\right)$ such that

$\nabla \bar{u}(h, \bar{m}(h)) \rightarrow H$ weakly in $L^{2}\left(\mathbb{R}^{3} ; \mathbb{R}^{3}\right)$ and $\frac{1}{h} \bar{u}(h, \bar{m}(h))_{, 3} \rightarrow w$ weakly in $L^{2}\left(\mathbb{R}^{3}\right)$. 
The $L^{p}$ version of the Poincaré Lemma implies the existence of $u(0) \in H_{l o c}^{1}\left(\mathbb{R}^{3} ; \mathbb{R}^{3}\right)$ such that $H=\nabla u(0)$. Thus,

$$
\nabla \bar{u}(h, \bar{m}(h)) \rightarrow \nabla u(0) \text { weakly in } L^{2}\left(\mathbb{R}^{3} ; \mathbb{R}^{3}\right),
$$

with $u(0)_{, 3}=0$, which implies that $\nabla u(0)=0$ in $L^{2}\left(\mathbb{R}^{3}\right)$ since $\nabla u(0) \in L^{2}\left(\mathbb{R}^{3} ; \mathbb{R}^{3}\right)$. Consequently,

$$
u(0)=0,
$$

since $u(0) \in U$. Let us show that the convergences in (4.4) and (4.5) are actually strong. Equation (4.3) reads in this case

$$
\int_{\mathbb{R}^{3}}\left|A_{h}^{T} \nabla_{h} \bar{u}(h, \bar{m}(h))\right|^{2} d_{h} d x=\int_{\mathbb{R}^{3}} A_{h}^{T} \nabla_{h} \bar{u}(h, \bar{m}(h)) \cdot \bar{m}(h) d_{h} d x .
$$

The convergences (4.4), (4.5) and the strong convergence of $\bar{m}(h)$ to $\bar{m}(0)$ in $L^{2}\left(\mathbb{R}^{3} ; \mathbb{R}^{3}\right)$ imply that

$$
\int_{\mathbb{R}^{3}} A_{h}^{T} \nabla_{h} \bar{u}(h, \bar{m}(h)) \cdot \bar{m}(h) d_{h} d x \rightarrow \int_{\Omega_{1}} A_{0}^{T}\left(\nabla_{p} u(0)+w e_{3}\right) \cdot \bar{m}(0) d_{0} d x,
$$

where $\nabla_{p} u=\left(\begin{array}{c}u_{, 1} \\ u_{, 2} \\ 0\end{array}\right)$. Thus, the $L^{2}\left(\mathbb{R}^{3} ; \mathbb{R}^{3}\right)$ norm of $A_{h}^{T} \nabla_{h} \bar{u}(h, \bar{m}(h))$ is convergent. In the next step, we identify the limit of this norm using some test functions constructed on the model of the one used by Gioia and James in [8]. We consider a sequence of functions $w^{\varepsilon} \in C_{c}^{\infty}\left(\mathbb{R}^{3}\right)$ that converges strongly in $L^{2}\left(\mathbb{R}^{3}\right)$ to $w$ when $\varepsilon \rightarrow 0$. Let $a_{\varepsilon}>0$ be such that the projection of the support of $w^{\varepsilon}$ on the $x_{3}$ axis belongs to $\left[-a_{\varepsilon}, a_{\varepsilon}\right]$ and we suppose that $a_{\varepsilon}>1$. We set for $\lambda>0$

$v_{h}^{\varepsilon, \lambda}\left(x_{1}, x_{2}, x_{3}\right)=h \int_{-a_{\varepsilon}}^{x_{3}} w^{\varepsilon}\left(x_{1}, x_{2}, s\right) d s-\frac{h}{\lambda} \int_{a_{\varepsilon}}^{x_{3}} \chi_{\left[a_{\varepsilon}, a_{\varepsilon}+\lambda\right]}(r) d r \int_{-a_{\varepsilon}}^{a_{\varepsilon}} w^{\varepsilon}\left(x_{1}, x_{2}, s\right) d s+c^{\varepsilon}$.

The constant $c^{\varepsilon}$ is chosen in such a way as to satisfy the condition on the ball $B$ and the second term guarantees that $\nabla v_{h}^{\varepsilon, \lambda} \in L^{2}\left(\mathbb{R}^{3} ; \mathbb{R}^{3}\right)$. We have that $\lim _{h \rightarrow 0} v_{h, 1}^{\varepsilon, \lambda}=$ $\lim _{h \rightarrow 0} v_{h, 2}^{\varepsilon, \lambda}=0$ in $L^{2}\left(\mathbb{R}^{3}\right)$ for $\varepsilon, \lambda$ fixed and

$$
\frac{1}{h} v_{h, 3}^{\varepsilon, \lambda}=w^{\varepsilon}-\frac{1}{\lambda} \chi_{\left[a_{\varepsilon}, a_{\varepsilon}+\lambda\right]}\left(x_{3}\right) \int_{-a_{\varepsilon}}^{a_{\varepsilon}} w^{\varepsilon}\left(x_{1}, x_{2}, s\right) d s .
$$

Since $\bar{u}(h, \bar{m}(h))$ is the solution of the minimization problem (4.1) we have that

$$
\begin{aligned}
\int_{\mathbb{R}^{3}}\left|A_{h}^{T} \nabla_{h} \bar{u}(h, \bar{m}(h))\right|^{2} & -2 A_{h}^{T} \nabla_{h} \bar{u}(h, \bar{m}(h)) \cdot \bar{m}(h) d_{h} d x \\
\leq & \int_{\mathbb{R}^{3}}\left|A_{h}^{T} \nabla_{h} v_{h}^{\varepsilon, \lambda}\right|^{2}-2 A_{h}^{T} \nabla_{h} v_{h}^{\varepsilon, \lambda} \cdot \bar{m}(h) d_{h} d x .
\end{aligned}
$$


Using (4.8), (4.6) and the fact that $A_{0}^{T}=\left(\nabla \Psi^{-1} \circ \Psi\left(x_{1}, x_{2}, 0\right)\right)^{T}=\left(a^{1}\left|a^{2}\right| a^{3}\right)$ in $\Omega_{1}$, where $a^{i}$ represents the vectors of the contravariant basis associated with the diffeomorphism $\psi$ with $\left|a^{3}\right|=1$, we obtain

$\int_{\mathbb{R}^{3}} A_{h}^{T} \nabla_{h} \bar{u}(h, \bar{m}(h)) \cdot \bar{m}(h) d_{h} d x \rightarrow \int_{\Omega_{1}} A_{0}^{T}\left(w e_{3}\right) \cdot \bar{m}(0) d_{0} d x=\int_{\Omega_{1}} w a^{3} \cdot \bar{m}(0) d_{0} d x$.

On the other hand, we have

$$
A_{h}(x)=\left(\nabla \Psi^{-1} \circ \Psi\left(x_{1}, x_{2}, h x_{3}\right)\right)^{T} \rightarrow\left(\nabla \Psi^{-1} \circ \Psi\left(x_{1}, x_{2}, 0\right)\right)^{T}=A_{0}^{T}(x),
$$

in $C^{0}\left(\mathbb{R}^{3}\right)$. Let $\widetilde{a^{i}}$ be the column vectors of $A_{0}^{T}$ which are defined in $\mathbb{R}^{3}$. We know that $\widetilde{a^{i}}=a^{i}$, in $\Omega_{1}$. Passing to the limit in (4.9) when $h \rightarrow 0$ and using (4.10), we obtain that

$$
\begin{aligned}
& \lim _{h \rightarrow 0} \int_{\mathbb{R}^{3}}\left|A_{h}^{T} \nabla_{h} \bar{u}(h, \bar{m}(h))\right|^{2} d_{h} d x \\
& \quad \leq \int_{\mathbb{R}^{3}}\left|\left(w^{\varepsilon}-\frac{1}{\lambda} \chi_{\left[a_{\varepsilon}, a_{\varepsilon}+\lambda\right]}\left(x_{3}\right) \int_{-a_{\varepsilon}}^{a_{\varepsilon}} w^{\varepsilon}\left(x_{1}, x_{2}, s\right) d s\right) \widetilde{a}^{3}\right|^{2} d_{0} d x \\
& -2 \int_{\Omega_{1}}\left\{\left(w^{\varepsilon}-\frac{1}{\lambda}\left(\chi_{\left[a_{\varepsilon}, a_{\varepsilon}+\lambda\right]}\left(x_{3}\right) \int_{-a_{\varepsilon}}^{a_{\varepsilon}} w^{\varepsilon}\left(x_{1}, x_{2}, s\right) d s\right)\right) a^{3} \cdot \bar{m}(0)+2 w a^{3} \cdot \bar{m}(0)\right\} d_{0} d x .
\end{aligned}
$$

Expanding this expression, we obtain

$$
\begin{aligned}
\lim _{h \rightarrow 0} \int_{\mathbb{R}^{3}} \mid & \left.A_{h}^{T} \nabla_{h} \bar{u}(h, \bar{m}(h))\right|^{2} d_{h} d x \\
& \leq \int_{\mathbb{R}^{3}}\left(\left|w^{\varepsilon}\right|^{2}+\frac{1}{\lambda^{2}}\left|\chi_{\left[a_{\varepsilon}, a_{\varepsilon}+\lambda\right]}\left(x_{3}\right) \int_{-a_{\varepsilon}}^{a_{\varepsilon}} w^{\varepsilon}\left(x_{1}, x_{2}, s\right) d s\right|^{2}\right. \\
& \left.-\frac{2}{\lambda}\left(w^{\varepsilon} \chi_{\left[a_{\varepsilon}, a_{\varepsilon}+\lambda\right]}\left(x_{3}\right) \int_{-a_{\varepsilon}}^{a_{\varepsilon}} w^{\varepsilon}\left(x_{1}, x_{2}, s\right) d s\right)\right)\left|\widetilde{a^{3}}\right|^{2} d_{0} d x \\
& +\int_{\Omega_{1}}\left\{\frac{2}{\lambda}\left(\chi_{\left[a_{\varepsilon}, a_{\varepsilon}+\lambda\right]}\left(x_{3}\right) \int_{0}^{a_{\varepsilon}} w^{\varepsilon}\left(x_{1}, x_{2}, s\right) d s\right) a^{3} \cdot \bar{m}(0)\right. \\
& \left.-2 w^{\varepsilon} a^{3} \cdot \bar{m}(0)+2 w a^{3} \cdot \bar{m}(0)\right\} d_{0} d x .
\end{aligned}
$$

Since the projection of the support of $w^{\varepsilon}$ on the $x_{3}$-axis belongs to $\left[-a_{\varepsilon}, a_{\varepsilon}\right]$, we have that $w^{\varepsilon}(x) \chi_{\left[a_{\varepsilon}, a_{\varepsilon}+\lambda\right]}\left(x_{3}\right)=0$, a.e. in $\mathbb{R}^{3}$, thus the third term of the right hand side of inequality (4.11) vanishes. We also have that $\chi_{\left[a_{\varepsilon}, a_{\varepsilon}+\lambda\right]}\left(x_{3}\right)=0$ in $\Omega_{1}$ since $a_{\varepsilon}>1$. Thus, the fourth term of the right hand side of inequality (4.11) also 
vanishes. Considering all this, inequality (4.11) becomes

$$
\begin{aligned}
\lim _{h \rightarrow 0} \int_{\mathbb{R}^{3}}\left|A_{h}^{T} \nabla_{h} \bar{u}(h, \bar{m}(h))\right|^{2} d_{h} d x \\
\leq \int_{\mathbb{R}^{3}}\left(\left|w^{\varepsilon}\right|^{2}+\frac{1}{\lambda^{2}}\left|\chi_{\left[a_{\varepsilon}, a_{\varepsilon}+\lambda\right]}\left(x_{3}\right) \int_{-a_{\varepsilon}}^{a_{\varepsilon}} w^{\varepsilon}\left(x_{1}, x_{2}, s\right) d s\right|^{2}\right)\left|\widetilde{a}^{3}\right|^{2} d_{0} d x \\
\quad-2 \int_{\Omega_{1}}\left[w^{\varepsilon} a^{3} \cdot \bar{m}(0)-w a^{3} \cdot \bar{m}(0)\right] d_{0} d x \\
=\int_{\mathbb{R}^{3}}\left|w^{\varepsilon} \widetilde{a}^{3}\right|^{2} d_{0} d x-2 \int_{\Omega_{1}}\left[w^{\varepsilon} a^{3} \cdot \bar{m}(0)-w a^{3} \cdot \bar{m}(0)\right] d_{0} d x \\
\quad+\frac{1}{\lambda^{2}} \int_{\mathbb{R}^{2}}\left|\int_{-a_{\varepsilon}}^{a_{\varepsilon}} w^{\varepsilon}\left(x_{1}, x_{2}, s\right) d s\right|^{2}\left|\widetilde{a}^{3}\right|^{2} d_{0} d \sigma\left(\int_{\mathbb{R}} \chi_{\left[a_{\varepsilon}, a_{\varepsilon}+\lambda\right]}^{2}\left(x_{3}\right) d x_{3}\right) .
\end{aligned}
$$

Noting that $\int_{\mathbb{R}} \chi_{\left[a_{\varepsilon}, a_{\varepsilon}+\lambda\right]}^{2}(x) d x=\lambda$, and passing to the limit when $\lambda \rightarrow+\infty$ we obtain

$$
\begin{aligned}
\lim _{h \rightarrow 0} \int_{\mathbb{R}^{3}}\left|A_{h}^{T} \nabla_{h} \bar{u}(h, \bar{m}(h))\right|^{2} d_{h} d x & \\
& \leq \int_{\mathbb{R}^{3}}\left|w^{\varepsilon} \widetilde{a}^{3}\right|^{2} d_{0} d x-2 \int_{\Omega_{1}} w^{\varepsilon} a^{3} \cdot \bar{m}(0)-w a^{3} \cdot \bar{m}(0) d_{0} d x .
\end{aligned}
$$

Then we take the limit as $\varepsilon \rightarrow 0$ and obtain

$$
\lim _{h \rightarrow 0} \int_{\mathbb{R}^{3}}\left|A_{h}^{T} \nabla_{h} \bar{u}(h, \bar{m}(h))\right|^{2} d_{h} d x \leq \int_{\mathbb{R}^{3}}\left|w \widetilde{a}^{3}\right|^{2} d_{0} d x .
$$

Due to the weak convergences in (4.4), (4.5) and since $A_{0}^{T}\left(\nabla_{p} u(0)+w e_{3}\right)=$ $A_{0}^{T}\left(w e_{3}\right)=w \widetilde{a}^{3}$ in $\mathbb{R}^{3}$, we have

$$
\int_{\mathbb{R}^{3}}\left|w \widetilde{a}^{3}\right|^{2} d_{0} d x \leq \liminf \int_{\mathbb{R}^{3}}\left|A_{h}^{T} \nabla_{h} \bar{u}(h, \bar{m}(h))\right|^{2} d_{h} d x .
$$

Then, we deduce the convergence of the norm thanks to (4.12) and (4.13), therefore

$$
A_{h}^{T} \nabla_{h} \bar{u}(h, \bar{m}(h)) \rightarrow w \widetilde{a}^{3},
$$

in $L^{2}\left(\mathbb{R}^{3} ; \mathbb{R}^{3}\right)$. It follows that

$$
\nabla_{h} \bar{u}(h, \bar{m}(h))=A_{h}{ }^{-T} A_{h}^{T} \nabla_{h} \bar{u}(h, \bar{m}(h)) \rightarrow A_{0}{ }^{-T} A_{0}^{T}\left(w e_{3}\right)=w e_{3},
$$

that gives the strong convergence in (4.4) and (4.5). Let us now identify the norm of $w \widetilde{a}^{3}$ in $L^{2}\left(\mathbb{R}^{3}\right)$. Letting $h \rightarrow 0$ in (4.7), we get

$$
\int_{\mathbb{R}^{3}}\left|w \widetilde{a}^{3}\right|^{2} d_{0} d x=\int_{\Omega_{1}} w a_{3} \cdot \bar{m}(0) d_{0} d x .
$$


We set

$\xi_{h}^{\varepsilon}=h \int_{-1}^{x_{3}}\left(\widetilde{a}^{3} \cdot \bar{m}^{\varepsilon}(0)\right)\left(x_{1}, x_{2}, s\right) d s-\frac{h}{\lambda} \int_{1}^{x_{3}} \chi_{[1,1+\lambda]}(r) d r \int_{-1}^{1}\left(\widetilde{a}^{3} \cdot \bar{m}^{\varepsilon}(0)\right)\left(x_{1}, x_{2}, s\right) d s+c^{\varepsilon}$,

where $\bar{m}^{\varepsilon}(0)$ is a sequence in $C_{0}^{\infty}\left(\mathbb{R}^{3} ; \mathbb{R}^{3}\right)$ that converges to $\bar{m}(0)$ in $L^{2}\left(\mathbb{R}^{3} ; \mathbb{R}^{3}\right)$ when $\varepsilon$ goes to zero with $\operatorname{supp} \bar{m}^{\varepsilon}(0) \subset \Omega_{1}$ and $c^{\varepsilon}$ a constant depending on $\varepsilon$. We have that $\xi_{h}^{\varepsilon} \in U$, with

$$
\frac{1}{h} \xi_{h, 3}^{\varepsilon}=\widetilde{a}^{3} \cdot \bar{m}^{\varepsilon}(0)-\frac{1}{\lambda} \chi_{[1,1+\lambda]}\left(x_{3}\right) \int_{-1}^{1}\left(\widetilde{a}^{3} \cdot \bar{m}^{\varepsilon}(0)\right)\left(x_{1}, x_{2}, s\right) d s .
$$

The minimization problem (4.1) implies that

$$
\int_{\mathbb{R}^{3}}\left|A_{h}^{T} \nabla_{h} \bar{u}(h, \bar{m}(h))-\bar{m}(h)\right|^{2} d_{h} d x \leq \int_{\mathbb{R}^{3}}\left|A_{h}^{T} \nabla_{h} \xi_{h}^{\varepsilon}-\bar{m}(h)\right|^{2} d_{h} d x .
$$

Letting $h$ go to zero, we see that

$$
\begin{aligned}
\int_{\mathbb{R}^{3}} \mid & A_{0}^{T}\left(w e_{3}\right)-\left.\bar{m}(0)\right|^{2} d_{0} d x \leq \int_{\mathbb{R}^{3}} \mid A_{0}^{T}\left(\left(\widetilde{a}^{3} \cdot \bar{m}^{\varepsilon}(0)\right.\right. \\
& \left.\left.\quad-\frac{1}{\lambda} \chi_{[1,1+\lambda]}\left(x_{3}\right) \int_{-1}^{1}\left(\widetilde{a}^{3} \cdot \bar{m}^{\varepsilon}(0)\right)\left(x_{1}, x_{2}, s\right) d s\right) e_{3}\right)-\left.\bar{m}(0)\right|^{2} d_{0} d x .
\end{aligned}
$$

Let us estimate the right-hand side of this enequality, we have that

$$
\begin{gathered}
\int_{\mathbb{R}^{3}}\left|A_{0}^{T}\left(\left(\widetilde{a}^{3} \cdot \bar{m}^{\varepsilon}(0)-\frac{1}{\lambda} \chi_{[1,1+\lambda]}\left(x_{3}\right) \int_{-1}^{1}\left(\widetilde{a}^{3} \cdot \bar{m}^{\varepsilon}(0)\right)\left(x_{1}, x_{2}, s\right) d s\right) e_{3}\right)-\bar{m}(0)\right|^{2} d_{0} d x \\
=\int_{\mathbb{R}^{3}}\left|\left(\widetilde{a}^{3} \cdot \bar{m}^{\varepsilon}(0)-\frac{1}{\lambda} \chi_{[1,1+\lambda]}\left(x_{3}\right) \int_{-1}^{1}\left(\widetilde{a}^{3} \cdot \bar{m}^{\varepsilon}(0)\right)\left(x_{1}, x_{2}, s\right) d s\right) \widetilde{a}^{3}-\bar{m}(0)\right|^{2} d_{0} d x \\
=\int_{\mathbb{R}^{3}}\left|\widetilde{a}^{3} \cdot \bar{m}^{\varepsilon}(0)\right|^{2}+\frac{1}{\lambda^{2}} \chi_{[1,1+\lambda]}^{2}\left(x_{3}\right)\left|\int_{-1}^{1}\left(\widetilde{a}^{3} \cdot \bar{m}^{\varepsilon}(0)\right)\left(x_{1}, x_{2}, s\right) d s\right|^{2}\left|\widetilde{a}^{3}\right|^{2} \\
\quad+|\bar{m}(0)|^{2}-\frac{2}{\lambda}\left(\widetilde{a}^{3} \cdot \bar{m}^{\varepsilon}(0) \chi_{[1,1+\lambda]}\left(x_{3}\right) \int_{-1}^{1}\left(\widetilde{a}^{3} \cdot \bar{m}^{\varepsilon}(0)\right)\left(x_{1}, x_{2}, s\right) d s\right) \\
-2\left(\left(\widetilde{a}^{3} \cdot \bar{m}^{\varepsilon}(0)\right) \widetilde{a}^{3} \cdot \bar{m}(0)\right)-\frac{2}{\lambda}\left(\chi_{[1,1+\lambda]}\left(x_{3}\right) \int_{-1}^{1}\left(\widetilde{a}^{3} \cdot \bar{m}^{\varepsilon}(0)\right)\left(x_{1}, x_{2}, s\right) d s \widetilde{a}^{3} \cdot \bar{m}(0)\right) d_{0} d x
\end{gathered}
$$

and since the projection of the support of $\bar{m}^{\varepsilon}(0)$ and of $\bar{m}(0)$ on the $x_{3}$ axis stays in $[0,1]$, it follows that

$$
\begin{aligned}
& \int_{\mathbb{R}^{3}} \frac{1}{\lambda}\left(\left(\widetilde{a}^{3} \cdot \bar{m}^{\varepsilon}(0)\right) \chi_{[1,1+\lambda]}\left(x_{3}\right) \int_{-1}^{1}\left(\widetilde{a}^{3} \cdot \bar{m}^{\varepsilon}(0)\right)\left(x_{1}, x_{2}, s\right) d s\right) d_{0} d x \\
& \quad=\int_{\mathbb{R}^{3}} \frac{1}{\lambda}\left(\left(\chi_{[1,1+\lambda]}\left(x_{3}\right) \int_{-1}^{1}\left(\widetilde{a}^{3} \cdot \bar{m}^{\varepsilon}(0)\right)\left(x_{1}, x_{2}, s\right) d s\right) \tilde{a}^{3} \cdot \bar{m}(0)\right) d_{0} d x=0 .
\end{aligned}
$$


We also have that

$$
\begin{aligned}
\frac{1}{\lambda^{2}} \int_{\mathbb{R}^{3}} \chi_{[1,1+\lambda]}^{2}\left(x_{3}\right) \mid & \left.\int_{-1}^{1}\left(\widetilde{a}^{3} \cdot \bar{m}^{\varepsilon}(0)\right)\left(x_{1}, x_{2}, s\right) d s\right|^{2}\left|\widetilde{a}^{3}\right|^{2} d_{0} d x \\
& =\frac{1}{\lambda} \int_{\mathbb{R}^{2}}\left|\int_{-1}^{1}\left(\widetilde{a}^{3} \cdot \bar{m}^{\varepsilon}(0)\right)\left(x_{1}, x_{2}, s\right) d s\right|^{2}\left|\widetilde{a}^{3}\right|^{2} d_{0} d \sigma \\
& 0 \\
\lambda \rightarrow+\infty & 0
\end{aligned}
$$

Applying all this in (4.15) we get, letting $\lambda \rightarrow \infty$

$$
\int_{\mathbb{R}^{3}}\left|w \widetilde{a}^{3}-\bar{m}(0)\right|^{2} d_{0} d x \leq \int_{\mathbb{R}^{3}}\left|\left(\widetilde{a}^{3} \cdot \bar{m}^{\varepsilon}(0)\right) \widetilde{a}^{3}-\bar{m}(0)\right|^{2} d_{0} d x
$$

Then, we let $\varepsilon \rightarrow 0$ and obtain

$$
\int_{\mathbb{R}^{3}}\left[\left|w \widetilde{a}^{3}\right|^{2}-2 w\left(\widetilde{a}^{3} \cdot \bar{m}(0)\right)\right] d_{0} d x \leq \int_{\mathbb{R}^{3}}\left[\left|\left(\widetilde{a}^{3} \cdot \bar{m}(0)\right)\right|^{2}-2\left|\left(\widetilde{a}^{3} \cdot \bar{m}(0)\right)\right|^{2}\right] d_{0} d x,
$$

and using (4.14) we get

$$
-\int_{\mathbb{R}^{3}} w\left(\widetilde{a}^{3} \cdot \bar{m}(0)\right) d_{0} d x \leq-\int_{\mathbb{R}^{3}}\left|\left(\widetilde{a}^{3} \cdot \bar{m}(0)\right)\right|^{2} d_{0} d x .
$$

Consequently, we have

$$
\begin{aligned}
\int_{\mathbb{R}^{3}}\left|\left(\widetilde{a}^{3} \cdot \bar{m}(0)\right)\right|^{2} d_{0} d x & \leq \int_{\mathbb{R}^{3}} w\left(\widetilde{a}^{3} \cdot \bar{m}(0)\right) d_{0} d x \\
& =\int_{\mathbb{R}^{3}}\left|w \widetilde{a}^{3}\right|^{2} d_{0} d x .
\end{aligned}
$$

Thanks to the Cauchy-Schwartz inequality, equation (4.14) gives

$$
\begin{aligned}
\int_{\mathbb{R}^{3}}\left|w \widetilde{a}^{3}\right|^{2} d_{0} d x & =\int_{\mathbb{R}^{3}}\left(w \tilde{a}^{3} \cdot \bar{m}(0)\right) d_{0} d x \\
& \leq\left(\int_{\mathbb{R}^{3}}|w|^{2} d_{0} d x\right)^{\frac{1}{2}}\left(\int_{\mathbb{R}^{3}}\left|\left(\widetilde{a}^{3} \cdot \bar{m}(0)\right)\right|^{2} d_{0} d x\right)^{\frac{1}{2}},
\end{aligned}
$$

thus,

$$
\left(\int_{\mathbb{R}^{3}}\left|w \widetilde{a}^{3}\right|^{2} d_{0} d x\right)^{\frac{1}{2}} \leq\left(\int_{\mathbb{R}^{3}}\left|\left(\widetilde{a}^{3} \cdot \bar{m}(0)\right)\right|^{2} d_{0} d x\right)^{\frac{1}{2}},
$$

and using (4.17) we obtain

$$
\begin{aligned}
\left(\int_{\mathbb{R}^{3}}\left|w \tilde{a}^{3}\right|^{2} d_{0} d x\right)^{\frac{1}{2}} & =\left(\int_{\mathbb{R}^{3}}\left|\left(\widetilde{a}^{3} \cdot \bar{m}(0)\right)\right|^{2} d_{0} d x\right)^{\frac{1}{2}} \\
& =\left(\int_{\Omega_{1}}\left|\left(a_{3} \cdot \bar{m}(0)\right)\right|^{2} d_{0} d x\right)^{\frac{1}{2}} .
\end{aligned}
$$

Finally, we have

$$
E_{\mathrm{mag}}(h)(\bar{m}(h)) \rightarrow E_{\mathrm{mag}}(0)(\bar{m}(0))=\frac{1}{2} \int_{\Omega_{1}}\left|\left(a_{3} \cdot \bar{m}(0)\right)\right|^{2} d_{0} d x .
$$


Now that we know the behavior of the magnetostatic energy term, we can compute the $\Gamma$-limit of the free energy $e(h)$.

\section{$5 \quad \Gamma$-convergence and behavior of minimizers}

We are interested in the behavior of the diagonal minimizing sequence $m(h)$ of the energy $e(h)$, that verifies

$$
m(h) \in V \text { and } e(h)(m(h))=\inf _{m \in V} e(h)(m)+\varepsilon(h),
$$

with $\varepsilon(h) \rightarrow 0$ when $h \rightarrow 0$ and

$$
\begin{aligned}
& V=\left\{m \in L^{\infty}\left(\mathbb{R}^{3} ; \mathbb{R}^{3}\right), \text { such that } m_{\mid \Omega_{1}} \in W^{1, p}\left(\Omega_{1} ; \mathbb{R}^{3}\right),\right. \\
& \left.\qquad|m|=1 \text {, a.e. in } \Omega_{1} \text { and } m=0 \text {, a.e. in } \Omega_{1}^{c}\right\} .
\end{aligned}
$$

In the following, we will identify functions in $V$ with functions in $W^{1, p}\left(\Omega_{1} ; S^{2}\right)$ while keeping the same notation.

Let us begin by a lemma on deformations with bounded energies.

Lemma 5.1 Let $m(h)$ be a sequence of magnetizations in V verifying $e(h)(m(h)) \leq$ c. Then, we have

$$
\|\nabla m(h)\|_{L^{p}\left(\Omega_{1} ; \mathbb{R}^{3}\right)} \leq c \text { and } \frac{1}{h}\left\|m(h)_{, 3}\right\|_{L^{p}\left(\Omega_{1} ; \mathbb{R}^{3}\right)} \leq c .
$$

Moreover, the limit points of this sequence for the weak topology of $W^{1, p}$ belong to the set

$$
V_{M}=\left\{m \in V \text { et } m_{, 3}=0 \text { in } \Omega_{1}\right\} .
$$

Proof Since the magnetostatic energy term is positive, we have

$$
\int_{\Omega_{1}} W\left(m(h),\left(m(h)_{, 1}, m(h)_{, 2}, \frac{1}{h} m(h)_{, 3}\right) A_{h}\right) d_{h} d x \leq c,
$$

and (3.2) implies

$$
\left\|\left(m(h)_{, 1}, m(h)_{, 2}, \frac{1}{h} m(h)_{, 3}\right) A_{h}\right\|_{L^{p}\left(\Omega_{1} ; \mathbb{R}^{3}\right)} \leq c .
$$

Thus, we obtain (5.1). We deduce the existence of a subsequence, still denoted $h$ and $m(0) \in W^{1, p}\left(\Omega_{1} ; \mathbb{R}^{3}\right)$ satisfying

$$
m(h) \rightarrow m(0) \text { in } W^{1, p}\left(\Omega_{1} ; \mathbb{R}^{3}\right)
$$

with $m(0)_{, 3}=0$. The weak convergence in $W^{1, p}\left(\Omega_{1} ; \mathbb{R}^{3}\right)$ implies that for a subsequence still denoted $h$ we have $m(h) \rightarrow m(0)$ in $L^{p}\left(\Omega_{1} ; \mathbb{R}^{3}\right)$, and thus, for a subsequence, we have $m(h) \rightarrow m(0)$ a.e. in $\Omega_{1}$, which implies that for almost every $x \in \Omega_{1}$ we have $|m(0)|=1$. 
Before announcing the main result of this section, let us recall the definition of tangential quasiconvexity, a notion introduced by B. Dacorogna, I. Fonseca, J. Maly and K. Trivisa in [5] that gives the relaxation of a class of functionals where admissible deformations are constrained to remain in a $C^{1}$ submanifold of dimension $q$ of $\mathbb{R}^{d}$. This definition was generalized by R. Alicandro and C. Leone in [2] in the case of functionals depending on the gradient of the deformation and the deformation itself. We denote by $M^{d \times N}$ the set of matrices $d \times N$. Let $f: M^{d \times N} \rightarrow\left[0,+\infty\left[\right.\right.$ be a Borel measurable function and $M$ a $C^{1}$ submanifold of dimension $q$ of $\mathbb{R}^{d}$. We denote by $T_{y}(M)$ the tangent space to $M$ at $y \in M$.

Definition 5.1 Let $y \in M$ and $\xi \in\left[T_{y}(M)\right]^{N}$. The tangential quasiconvexification of $f$ in $\xi$ relative to $y$ is defined by

$$
Q_{T}^{N, d} f(y, \xi):=\inf \left\{\int_{Q} f(y, \xi+\nabla \varphi(x)) d x: \varphi \in W_{0}^{1, \infty}\left(Q ; T_{y}(M)\right)\right\},
$$

with $Q$ a cube in $\mathbb{R}^{N}$.

In our case $M$ is the unit sphere $S^{2}$ of $\mathbb{R}^{3}$ and $T_{y}\left(S^{2}\right)=y^{\perp}$, the plane orthogonal to $y$. We recall two results proved in [5]

Proposition 5.1 Let $f: M^{d \times N} \rightarrow[0,+\infty)$ be a continuous function such that there exists $c>0$ and $p \geq 1$ verifying $0 \leq f(\xi) \leq c\left(1+|\xi|^{p}\right)$, for every $\xi \in M^{d \times N}$. We define the functional $J$ by

$$
J(u):=\int_{\Omega_{1}} Q_{T}^{N, d} f(u, \nabla u) d x, u \in W^{1, p}\left(\Omega_{1} ; M\right) .
$$

Then, $J($.$) is sequentially weakly lower semicontinuous in W^{1, p}\left(\Omega_{1} ; M\right)$.

Theorem 5.1 Let $f: M^{d \times N} \rightarrow[0,+\infty)$ a continuous function verifying $0 \leq f(\xi) \leq$ $c\left(1+|\xi|^{p}\right)$, for $p \geq 1, c>0$ and every $\xi \in M^{d \times N}$. If $M$ is a $C^{1}$ submanifold of dimension $q$, then

$$
F(u)=\int_{\Omega_{1}} Q_{T}^{N, d} f(u, \nabla u) d x
$$

where

$$
F(u):=\inf _{u_{n}}\left\{\liminf _{n \rightarrow+\infty} \int_{\Omega_{1}} f\left(\nabla u_{n}\right) d x: u_{n} \rightarrow u \operatorname{in} W^{1, p}\left(\Omega_{1} ; M\right)\right\} .
$$

In our case, the magnetization $m$ is constrained to remain in the sphere $S^{2}$. Consequently, $m_{, i}$ will be in $m^{\perp}$ almost everywhere. As in Acerbi, Buttazzo and Percivale [1] for nonlinearly elastic strings and Le Dret and Raoult [10] for membranes, 
we define $W_{0}: \Omega_{1} \times \mathbb{R}^{3} \times M^{3 \times 2} \rightarrow \mathbb{R}$ by setting

$$
\begin{aligned}
W_{0}(x, y, \bar{F}) & :=\inf _{z \in y^{\perp}} W\left(y,(\bar{F} \mid z) A_{0}(x)\right) \\
& =\inf _{z \in \mathbb{R}^{3}} \bar{W}\left(y,(\bar{F} \mid z) A_{0}(x)\right),
\end{aligned}
$$

with $A_{0}(x)=\nabla \Psi^{-1} \circ \Psi\left(x_{1}, x_{2}, 0\right)$ and

$$
\bar{W}\left(y,(\bar{F} \mid z) A_{0}(x)\right)= \begin{cases}W\left(y,(\bar{F} \mid z) A_{0}(x)\right), & \text { si } z \in y^{\perp} \\ +\infty, & \text { otherwise. }\end{cases}
$$

We show as in Le Dret and Raoult [10], that the function $W_{0}$ is continuous and satisfies properties analogous to those verified by $W$, that is to say

$$
\left\{\begin{array}{l}
\exists c^{\prime}>0, \forall \bar{F} \in M^{3,2}, \forall y \in \mathbb{R}^{3}, \forall x \in \bar{\omega},\left|W_{0}(x, y, \bar{F})\right| \leq c^{\prime}\left(1+|\bar{F}|^{p}\right), \\
\exists \gamma^{\prime}>0, \exists \beta^{\prime} \geq 0, \forall \bar{F} \in M^{3,2}, \forall y \in \mathbb{R}^{3}, \forall x \in \bar{\omega}, W_{0}(x, y, \bar{F}) \geq \gamma^{\prime}|\bar{F}|^{p}-\beta^{\prime} .
\end{array}\right.
$$

In order to study the behavior of the free energy of the ferromagnetic curved film and its possible minimizers when the thickness of the film goes to zero, we are going to compute its $\Gamma$-limit. The natural space in which we would like to compute the $\Gamma$-limit is $W^{1, p}\left(\Omega_{1} ; \mathbb{R}^{3}\right)$. However, $W^{1, p}\left(\Omega_{1} ; \mathbb{R}^{3}\right)$ endowed with the weak topology is not metrizable. Thus, we extend the energy $e(h)$ to all $L^{p}\left(\Omega_{1} ; \mathbb{R}^{3}\right)$ by setting

$$
\text { for all } m \in L^{p}\left(\Omega_{1} ; \mathbb{R}^{3}\right), e^{*}(h)(m)= \begin{cases}e(h)(m) & \text { if } m \in V, \\ +\infty & \text { otherwise }\end{cases}
$$

We now are in a position to compute the $\Gamma$-limit of the free energy.

Theorem 5.2 The sequence of energies $e^{*}(h) \Gamma$-converges for the strong topology of $L^{p}\left(\Omega_{1} ; \mathbb{R}^{3}\right)$ to

$$
e^{*}(0)(m)= \begin{cases}\int_{\omega} Q_{T}^{2,3} W_{0}\left(x, m(x),\left(m_{, 1} \mid m_{, 2}\right)\right)+\left|\left(a_{3}(x), m\right)\right|^{2} d_{0} d x & \text { if } m \in V_{M} \\ +\infty & \text { otherwise } .\end{cases}
$$

The proof of the theorem follows from the following propositions.

Proposition 5.2 We have

$$
\Gamma-\liminf e^{*}(h) \geq e^{*}(0) .
$$


Preuve We obtain the lower bound of the $\Gamma$-limit by showing that for any sequence $m^{h} \in L^{p}\left(\Omega_{1} ; \mathbb{R}^{3}\right)$ converging strongly to $m^{0}$ in $L^{p}\left(\Omega_{1} ; \mathbb{R}^{3}\right)$, we have

$$
\liminf e^{*}(h)\left(m^{h}\right) \geq e^{*}(0)\left(m^{0}\right) .
$$

First, if $m^{h} \notin V$, then $e^{*}(h)\left(m^{h}\right)=+\infty$ and the result is trivial. In the same way, if $m^{h} \in V$ and $\liminf e^{*}(h)\left(m^{h}\right)=+\infty$, the result is obvious.

Next, we consider $m^{h} \in V$, such that $\liminf e^{*}(h)\left(m^{h}\right)=\liminf e(h)\left(m^{h}\right)<$ $+\infty$. This implies that for a subsequence, there exists $c>0$ such that $e(h)\left(m^{h}\right)<c$. Using the lemma 5.1 we get that for a subsequence still denoted $h$ we have that $m^{h} \rightarrow m^{0}$ in $W^{1, p}\left(\Omega_{1} ; \mathbb{R}^{3}\right)$. Thanks to the fact that $m^{h}$ is uniformly bounded in $L^{\infty}\left(\Omega_{1} ; \mathbb{R}^{3}\right)$ we obtain that $m^{h} \rightarrow m^{0}$ in $L^{s}\left(\Omega_{1} ; \mathbb{R}^{3}\right)$, for all $s>1$, in particular for $s=2$. We thus deduce from proposition 4.1 that

$$
E_{\mathrm{mag}}(h)\left(\bar{m}^{h}\right) \rightarrow E_{\mathrm{mag}}(0)\left(\bar{m}^{0}\right)=\frac{1}{2} \int_{\Omega_{1}}\left|\left(a_{3}, \bar{m}^{0}\right)\right|^{2} d_{0} d x .
$$

Let us set

$$
E_{\mathrm{ea}}(h)(m)=\int_{\Omega_{1}} W\left(m,\left(m_{, 1}\left|m_{, 2}\right| \frac{1}{h} m_{, 3}\right) A_{h}\right) d_{h} d x,
$$

which represents the exchange and anisotropic energies. We have

$$
\begin{aligned}
& E_{\mathrm{ea}}(h)\left(m^{h}\right)=\int_{\Omega_{1}} W\left(m^{h},\left(m_{, 1}^{h}\left|m_{, 2}^{h}\right| \frac{1}{h} m_{, 3}^{h}\right) A_{h}\right) d_{h} d x \\
&=\int_{\Omega_{1}}\left\{W\left(m^{h},\left(m_{, 1}^{h}\left|m_{, 2}^{h}\right| \frac{1}{h} m_{, 3}^{h}\right) A_{0}\right)+R\left(x, h, m^{h}\right)\right\} d_{h} d x
\end{aligned}
$$

where

$$
R\left(x, h, m^{h}\right)=W\left(m^{h},\left(m_{, 1}^{h}\left|m_{, 2}^{h}\right| \frac{1}{h} m_{, 3}^{h}\right) A_{h}\right)-W\left(m^{h},\left(m_{, 1}^{h}\left|m_{, 2}^{h}\right| \frac{1}{h} m_{, 3}^{h}\right) A_{0}\right) .
$$

Using the properties (3.2) of $W$ and those of the diffeomorphism $\Psi$, we show as in [11] that

$$
\int_{\Omega_{1}} R\left(x, h, m^{h}\right) d_{h} d x \rightarrow 0 \text { when } h \rightarrow 0 \text {. }
$$

Thus, we have

$$
\begin{aligned}
E_{\mathrm{ea}}(h)\left(m^{h}\right) \geq \int_{\Omega_{1}}\left\{W_{0}\left(x, m^{h},\left(m_{, 1}^{h} \mid m_{, 2}^{h}\right)\right)+R\left(x, h, m^{h}\right)\right\} d_{h} d x \\
\quad \geq \int_{\Omega_{1}}\left\{Q_{T}^{2,3} W_{0}\left(x, m^{h},\left(m_{, 1}^{h} \mid m_{, 2}^{h}\right)\right)+R\left(x, h, m^{h}\right)\right\} d_{h} d x .
\end{aligned}
$$


Taking the limit when $h \rightarrow 0$ we obtain using (5.4) and proposition(5.1) that

$$
\liminf E_{\mathrm{ea}}(h)\left(m^{h}\right) \geq \int_{\Omega_{1}} Q_{T}^{2,3} W_{0}\left(x, m^{0},\left(m_{, 1}^{0} \mid m_{, 2}^{0}\right)\right) d_{0} d x .
$$

Using (5.3) and since $m_{, 3}^{0}=0$ (lemma 5.1), we obtain that

$$
\liminf e(h)\left(m^{h}\right) \geq \int_{\omega}\left\{Q_{T}^{2,3} W_{0}\left(x, m^{0}(x),\left(m_{, 1}^{0} \mid m_{, 2}^{0}\right)\right)+\left|\left(a_{3}(x), m^{0}\right)\right|^{2}\right\} d_{0} d x,
$$

Thus, $\liminf e^{*}(h)\left(m^{h}\right) \geq e^{*}(0)\left(m^{0}\right)$ which implies that

$$
\Gamma-\liminf e^{*}(h) \geq e^{*}(0) .
$$

Then we proceed to the computation of the upper bound of the $\Gamma$-limit. We will need the following lemma (see [10])

Lemma 5.2 Let $X \hookrightarrow Y$ be two Banach spaces such that $X$ is reflexive and compactly embedded in $Y$. Let $G: X \rightarrow \mathbb{R}$ a function such that $\forall v \in X, G(v) \geq g\left(\|v\|_{X}\right)$, with $g$ verifying $g(t) \rightarrow+\infty$ when $t \rightarrow+\infty$. Let $G^{*}$ defined by $G^{*}(v)=G(v)$ if $v \in X, G^{*}(v)=+\infty$ otherwise. Let $\Gamma-G$ be the lower semicontinuous envelope of $G$ for the weak topology of $X$ and $\Gamma-G^{*}$ the lower semicontinuous envelope of $G^{*}$ for the strong topology of $Y$. Then $\Gamma-G^{*}=(\Gamma-G)^{*}$.

Proposition 5.3 We have

$$
\Gamma-\limsup e^{*}(h) \leq e^{*}(0) .
$$

Preuve In order to find an upper bound for the $\Gamma$-limit, we have to construct a sequence $m^{h} \in L^{p}\left(\Omega_{1} ; \mathbb{R}^{3}\right)$ that converges strongly to $m^{0}$ in $L^{p}\left(\Omega_{1} ; \mathbb{R}^{3}\right)$ verifying

$$
\lim e^{*}(h)\left(m^{h}\right) \leq e^{*}(0)\left(m^{0}\right) .
$$

Let $m^{0} \in L^{p}\left(\Omega_{1} ; \mathbb{R}^{3}\right)$. First, if $m^{0} \notin V_{M}$, we have $e^{*}(0)\left(m^{0}\right)=+\infty$. Setting, for all $h, m^{h}=m^{0}$ we get (5.6). Next, we consider $m^{0} \in V_{M}$ and we set $m^{h}(x)=$ $m^{0}\left(x_{1}, x_{2}\right)+h x_{3} \xi\left(x_{1}, x_{2}\right)$, with $\xi \in W_{0}^{1, p}\left(\omega ; \mathbb{R}^{3}\right)$ to be chosen later. We temporarily do not consider the saturation constraint $\left|m^{h}\right|=1$, we will treat it later. We have $m^{h} \rightarrow m^{0}$ strongly in $W^{1, p}\left(\Omega_{1} ; \mathbb{R}^{3}\right)$. Using the Lebesgue convergence theorem, we have that for a subsequence still denoted $h$

$$
\begin{aligned}
E_{\mathrm{ea}}(h)\left(m^{h}\right) & =\int_{\Omega_{1}} W\left(m^{h},\left(m_{, \alpha}^{0}+h x_{3} \xi_{, \alpha} \mid \xi\right) A_{h}\right) d_{h} d x \\
& \rightarrow \int_{\Omega_{1}} W\left(m^{0},\left(m_{, \alpha}^{0} \mid \xi\right) A_{0}\right) d_{0} d x .
\end{aligned}
$$


The function $\mu: \omega \times \mathbb{R}^{3} \rightarrow \mathbb{R}$ defined by $\mu(x, z)=\bar{W}\left(m^{0}(x),\left(m_{, \alpha}^{0}(x) \mid z\right) A_{0}(x)\right)$ is a Caratheodory function. Thus, the measurable selection lemma implies that, there exists $\xi: \omega \rightarrow \mathbb{R}^{3}$ measurable such that

$$
W_{0}\left(x, m^{0}(x),\left(m_{, 1}^{0}(x) \mid m_{, 2}^{0}(x)\right)\right)=\bar{W}\left(m^{0}(x),\left(m_{, \alpha}^{0}(x) \mid \xi(x)\right) A_{0}(x)\right),
$$

(see [7]). If $\xi \notin m^{0^{\perp}}$, this implies that $W_{0}\left(x, m^{0}(x),\left(m_{, 1}^{0}(x) \mid m_{, 2}^{0}(x)\right)\right)=+\infty$ and the result is obvious. If $\xi \in m^{0^{\perp}}$ a.e., this implies that

$$
\begin{aligned}
W_{0}\left(x, m^{0}(x),\left(m_{, 1}^{0}(x) \mid m_{, 2}^{0}(x)\right)\right) & =\bar{W}\left(m^{0}(x),\left(m_{, \alpha}^{0}(x) \mid \xi(x)\right) A_{0}(x)\right) \\
& =W\left(m^{0}(x),\left(m_{, \alpha}^{0}(x) \mid \xi(x)\right) A_{0}(x)\right) .
\end{aligned}
$$

and thanks to the properties (3.2) of $W$, we obtain that $\xi \in L^{p}\left(\omega ; \mathbb{R}^{3}\right)$. The density of $C_{c}^{\infty}\left(\omega ; \mathbb{R}^{3}\right)$ in $L^{p}\left(\omega ; \mathbb{R}^{3}\right)$ implies the existence of a sequence $\xi^{\varepsilon} \in C_{c}^{\infty}\left(\omega ; \mathbb{R}^{3}\right)$ verifying $\xi^{\varepsilon} \rightarrow \xi$ strongly in $L^{p}\left(\omega ; \mathbb{R}^{3}\right)$. Next, we project $\xi^{\varepsilon}$ on $m^{0^{\perp}}$ setting $\bar{\xi}^{\varepsilon}=\left(I-m^{0} \otimes m^{0}\right) \xi^{\varepsilon}$. We have $\bar{\xi}^{\varepsilon} \in W_{0}^{1, p}\left(\omega ; \mathbb{R}^{3}\right)$ verifying $\bar{\xi}^{\varepsilon} \rightarrow \xi$ strongly in $L^{p}\left(\omega ; \mathbb{R}^{3}\right)$. Thus, using the Lebesgue convergence theorem, we have that for a subsequence still denoted $\varepsilon$

$\int_{\omega} W\left(m^{0}(x),\left(m_{, \alpha}^{0}(x) \mid \bar{\xi}^{\varepsilon}(x)\right) A_{0}(x)\right) d_{0} d x \rightarrow \int_{\omega} W\left(m^{0}(x),\left(m_{, \alpha}^{0}(x) \mid \xi(x)\right) A_{0}(x)\right) d_{0} d x$.

This means that for every $\eta>0$, there exists an $\varepsilon(\eta)>0$ such that $\forall \varepsilon \leq \varepsilon(\eta)$ we have

$$
\int_{\omega} W\left(m^{0},\left(m_{, \alpha}^{0} \mid \bar{\xi}^{\varepsilon}\right) A_{0}\right) d_{0} d x \leq \int_{\omega} W\left(m^{0},\left(m_{, \alpha}^{0} \mid \xi\right) A_{0}\right) d_{0} d x+\eta
$$

and thanks to (5.7) we obtain that

$$
\int_{\omega} W\left(m^{0},\left(m_{, \alpha}^{0} \mid \bar{\xi}^{\varepsilon}\right) A_{0}\right) d_{0} d x \leq \int_{\omega} W_{0}\left(x, m^{0}(x),\left(m_{, 1}^{0}(x) \mid m_{, 2}^{0}(x)\right)\right) d_{0} d x+\eta \operatorname{meas}(\widetilde{S}) .
$$

Let us set $\bar{m}_{\varepsilon}^{h}=m^{0}+h x_{3} \bar{\xi}^{\varepsilon}$. We have that $\bar{m}_{\varepsilon}^{h} \in W^{1, p}\left(\Omega_{1} ; \mathbb{R}^{3}\right)$. We fix $\varepsilon>0$, for $h<\frac{1}{2\left\|\bar{\xi}^{\varepsilon}\right\|_{L^{\infty}\left(\omega ; \mathbb{R}^{3}\right)}}$ and since $\left|m^{0}\right|=1$, we have $\left|\bar{m}_{\varepsilon}^{h}\right|>\left|m^{0}\right|-h\left\|\bar{\xi}^{\varepsilon}\right\|_{L^{\infty}\left(\omega ; \mathbb{R}^{3}\right)}>\frac{1}{2}$, a.e.Then, we set $m_{\varepsilon}^{h}=\frac{\bar{m}_{\varepsilon}^{h}}{\left|\bar{m}_{\varepsilon}^{h}\right|}$. By construction, we have $m_{\varepsilon}^{h} \in V$ thanks to the algebra property of $W^{1, p}\left(\omega ; \mathbb{R}^{3}\right) \cap L^{\infty}\left(\omega ; \mathbb{R}^{3}\right)$. We have

$$
\nabla m_{\varepsilon}^{h}=\left(\frac{I}{\left|\bar{m}_{\varepsilon}^{h}\right|}-\frac{\bar{m}_{\varepsilon}^{h} \otimes \bar{m}_{\varepsilon}^{h}}{\left|\bar{m}_{\varepsilon}^{h}\right|^{3}}\right) \nabla \bar{m}_{\varepsilon}^{h} .
$$

We also have $m_{\varepsilon}^{h} \underset{h \rightarrow 0}{\rightarrow} m^{0}$ strongly in $L^{p}\left(\Omega_{1} ; \mathbb{R}^{3}\right)$, and

$$
m_{\varepsilon, \alpha}^{h} \underset{h \rightarrow 0}{\rightarrow}\left(I-m^{0} \otimes m^{0}\right) m_{, \alpha}^{0} \text { strongly in } L^{p}\left(\Omega_{1} ; \mathbb{R}^{3}\right) .
$$


On the other hand, we have

$$
\left(\left(m^{0} \otimes m^{0}\right) \nabla m^{0}\right)_{i, \alpha}=m_{i}^{0}\left(m^{0}, m_{, \alpha}^{0}\right)=0,
$$

since $\left|m^{0}\right|=1$, and we also have

$$
\frac{1}{h} m_{\varepsilon, 3}^{h} \underset{h \rightarrow 0}{\rightarrow}\left(I-m^{0} \otimes m^{0}\right) \bar{\xi}^{\varepsilon} \text { strongly in } L^{p}\left(\Omega_{1} ; \mathbb{R}^{3}\right) .
$$

Now, $\bar{\xi}^{\varepsilon} \in m^{0^{\perp}}$ so that we have $\left(m^{0} \otimes m^{0}\right) \bar{\xi}^{\varepsilon}=0$ and thus, we obtain

$$
m_{\varepsilon, \alpha}^{h} \underset{h \rightarrow 0}{\rightarrow} m_{, \alpha}^{0} \text { and } \frac{1}{h} m_{\varepsilon, 3}^{h} \underset{h \rightarrow 0}{\rightarrow} \bar{\xi}^{\varepsilon} \text { strongly in } L^{p}\left(\Omega_{1} ; \mathbb{R}^{3}\right)
$$

Using the Lebesgue theorem, we see that

$$
\begin{aligned}
E_{\mathrm{ea}}(h)\left(m_{\varepsilon}^{h}\right) & =\int_{\Omega_{1}} W\left(m_{\varepsilon}^{h},\left(m_{\varepsilon, \alpha}^{h} \mid \frac{1}{h} m_{\varepsilon, 3}^{h}\right) A_{h}\right) d_{h} d x \\
& \rightarrow \int_{h \rightarrow 0} W\left(m^{0},\left(m_{, \alpha}^{0} \mid \bar{\xi}^{\varepsilon}\right) A_{0}\right) d_{0} d x \\
& \leq \int_{\omega} W_{0}\left(x, m^{0}(x),\left(m_{, 1}^{0}(x) \mid m_{, 2}^{0}(x)\right)\right) d_{0} d x+\eta
\end{aligned}
$$

Thus, using proposition (4.1), we obtain

$$
\lim _{h \rightarrow 0} e(h)\left(m_{\varepsilon}^{h}\right) \leq \int_{\Omega_{1}}\left\{W_{0}\left(x, m^{0}(x),\left(m_{, 1}^{0}(x) \mid m_{, 2}^{0}(x)\right)\right)+\left|\left(a_{3}, m^{0}\right)\right|^{2}\right\} d_{0} d x+\eta .
$$

Let us set

$$
G^{*}(m)= \begin{cases}\int_{\omega} W_{0}\left(x, m(x),\left(m_{, 1} \mid m_{, 2}\right)\right)+\left|\left(a_{3}(x), m\right)\right|^{2} d_{0} d x & \text { if } m \in V_{M} \\ +\infty & \text { otherwise }\end{cases}
$$

We have shown that

$$
\lim _{h \rightarrow 0} e^{*}(h)\left(m_{\varepsilon}^{h}\right) \leq G^{*}\left(m^{0}\right)+\eta,
$$

which implies that $\Gamma-\lim \sup _{h \rightarrow 0} e^{*}(h)\left(m^{0}\right) \leq G^{*}\left(m^{0}\right)+\eta$. Since this is true for every $\eta>0$, we obtain that

$$
\Gamma-\limsup _{h \rightarrow 0} e^{*}(h)\left(m^{0}\right) \leq G^{*}\left(m^{0}\right) .
$$

We know that the $\Gamma$ - limsup of a function is weakly lower semicontinuous and that the lsc envelope of the function $G$ defined on $V_{M}$ by

$$
G(m)=\int_{\omega} W_{0}\left(x, m(x),\left(m_{, 1} \mid m_{, 2}\right)\right)+\left|\left(a_{3}(x), m\right)\right|^{2} d_{0} d x
$$


is $e(0)$ defined on $V_{M}$ by

$$
e(0)(m)=\int_{\omega} Q_{T}^{2,3} W_{0}\left(x, m(x),\left(m_{, 1} \mid m_{, 2}\right)\right)+\left|\left(a_{3}(x), m\right)\right|^{2} d_{0} d x,
$$

see [5]. Thus, using lemma 5.2, we obtain that the lsc envelope of $G^{*}$ is $e^{*}(0)$, and applying the lsc envelope to both sides of (5.8), we obtain that

$$
\Gamma-\limsup e^{*}(h) \leq e^{*}(0) .
$$

The conjunction of Propositions 5.2 and 5.3 gives Theorem 5.2.

Corollary 5.1 The diagonal minimizing sequence $m(h)$ of $e(h)$ is bounded in $\bar{V}$ and its limit points for the weak topology of $W^{1, p}\left(\Omega_{1}, \mathbb{R}^{3}\right)$ belong to $V_{M}$ and minimize the energy e $(0)$ defined by

$$
e(0)(m)=\int_{\omega}\left\{Q_{T}^{2,3} W_{0}\left(x, m(x),\left(m_{, 1} \mid m_{, 2}\right)\right)+\left|\left(a_{3}(x), m\right)\right|^{2}\right\} d_{0} d x .
$$

Preuve The proof of the corollary follows from Lemma 5.1 and the standard $\Gamma$ convergence argument.

\section{The curved two-dimensional limit model}

Since every magnetization $m$ in $V_{M}$ satisfies $m_{, 3}=0$ in $\Omega_{1}$, we will identify in the following magnetizations $m \in V_{M}$ with magnetizations $\bar{m} \in \bar{V}_{M}$ where

$\bar{V}_{M}=\left\{\bar{m} \in L^{p}\left(\mathbb{R}^{2} ; \mathbb{R}^{3}\right)\right.$, with $\bar{m}_{\mid \omega} \in W^{1, p}\left(\omega ; \mathbb{R}^{3}\right),|\bar{m}|=1$ a.e. in $\omega, \bar{m}=0$ a.e. in $\left.\omega^{c}\right\}$.

We consider another chart $\psi^{\prime}$ from $\omega^{\prime}$, an open set in $\mathbb{R}^{2}$ into $\widetilde{S}$. Working with this new chart, we obtain exactly the same $\Gamma$-convergence result, but this time, through another diffeomorphism $\Psi^{\prime}$. This means that the limit model is intrinsic and only depends on the curved surface $\widetilde{S}$. Let us thus write the limit model on the curved surface $\widetilde{S}$. As in [11], for any unit vector $e$ of $S^{2}$, we consider a bounded open set $O_{e} \subset e^{\perp}$ and denote by $\pi_{e}$ the orthogonal projection on this set. We extend any function $\chi \in W_{0}^{1, \infty}\left(O_{e}, \mathbb{R}^{3}\right)$ setting $\chi_{e}(y)=\chi\left(\pi_{e}(y)\right)$ and we define for any $y \in O_{e}$, $D_{e^{\perp}} \chi(y)=\nabla \chi_{e}(y)$. To any $m \in \bar{V}_{M}$ we associate a magnetization $\widetilde{m}: \mathbb{R}^{3} \rightarrow \mathbb{R}^{3}$ defined by $\widetilde{m}(\widetilde{x})=m\left(\psi^{-1}(\widetilde{x})\right)$. We have that $\widetilde{m} \in \widetilde{V}_{M}$ with

$$
\widetilde{V}_{M}=\left\{\widetilde{m} \in W^{1, p}\left(\Psi\left(\mathbb{R}^{2}\right) ; \mathbb{R}^{3}\right),|\widetilde{m}|=1 \text { a.e. in } \widetilde{S}, \widetilde{m}=0 \text { a.e. in } \widetilde{S}^{c} \text { et } \nabla \widetilde{m} \widetilde{n}(\widetilde{x})=0\right\} \text {, }
$$

where $\widetilde{n}$ denotes the normal vector to $\widetilde{S}$ (for a given orientation). All functions defined on the surface are implicitly assumed to be extended to a tubular neighborhood of the surface by being constant on each normal fiber. Partial derivatives are computed on these extensions and then restricted to the surface. 
Proposition 6.1 Every magnetization $\widetilde{m}$ associated with a magnetization m minimizing the limit energy e $(0)$, minimizes on $\widetilde{V}_{M}$ the energy $\widetilde{e}(0)$ defined by

$$
\widetilde{e}(0)(\widetilde{m})=\int_{\widetilde{S}}\left\{\widetilde{W}(\widetilde{n}(\widetilde{x}), \widetilde{m}, \nabla \widetilde{m}(\widetilde{x}))+|(\widetilde{n}(\widetilde{x}), \widetilde{m}(\widetilde{x}))|^{2}\right\} d \widetilde{x}
$$

where $\widetilde{W}: S^{2} \times S^{2} \times M^{3}$ is defined by

$$
\widetilde{W}(e, y, F)=\inf _{\chi \in W_{0}^{1, \infty}\left(O_{e} ; y^{\perp}\right)}\left[\frac{1}{\operatorname{meas}\left(O_{e}\right)} \int_{O_{e}}\left\{\inf _{z \in y^{\perp}} W\left(y, F+z \otimes e+D_{e^{\perp}} \chi(s)\right)\right\} d s\right] .
$$

Preuve We proceed as in Le Dret and Raoult [11]. For every $m \in V_{M}$ we have

$$
e(0)(m)=\int_{\omega} Q_{T}^{2,3} W_{0}\left(x, m(x),\left(m_{, 1} \mid m_{, 2}\right)\right)+\left|\left(a_{3}(x), m\right)\right|^{2} d_{0} d x .
$$

We set $\widetilde{x}=\psi(x), \widetilde{m}(\widetilde{x})=m(x)$. This implies that $\nabla m(x)=\nabla \widetilde{m}(\widetilde{x}) \nabla \psi(x)$ and setting $\widetilde{e}(0)(\widetilde{m})=e(0)(m)$ we obtain

$\widetilde{e}(0)(\widetilde{m})=\int_{\widetilde{S}}\left\{Q_{T}^{2,3} W_{0}\left(\psi^{-1}(\widetilde{x}), \widetilde{m}(\widetilde{x}), \nabla \widetilde{m}(\widetilde{x}) \nabla \psi\left(\psi^{-1}(\widetilde{x})\right)\right)+\left|\left(a_{3}\left(\psi^{-1}(\widetilde{x})\right), \widetilde{m}(\widetilde{x})\right)\right|^{2}\right\} d \widetilde{x}$

The integral representation of the tangential quasiconvex envelope (5.2) is written in our case

$$
Q_{T}^{2,3} W_{0}(x, y, \xi):=\inf \left\{\frac{1}{\operatorname{meas}(O)} \int_{O} W_{0}(x, y, \xi+\nabla \chi(s)) d s: \chi \in W_{0}^{1, \infty}\left(O ; y^{\perp}\right)\right\},
$$

with $O$ a bounded open domain in $\mathbb{R}^{3}$ and the infimum does not depend on the choice of this open domain. Thus, we have for all $\widetilde{x} \in \widetilde{S}$

$$
\begin{aligned}
& Q_{T}^{2,3} W_{0}\left(\psi^{-1}(\widetilde{x}), \widetilde{m}(\widetilde{x}), \nabla \widetilde{m}(\widetilde{x}) \nabla \psi\left(\psi^{-1}(\widetilde{x})\right)\right) \\
= & \inf _{\chi \in W_{0}^{1, \infty}\left(O ; \widetilde{m}(\widetilde{x})^{\perp}\right)} \frac{1}{\operatorname{meas}(O)} \int_{O} W_{0}\left(\psi^{-1}(\widetilde{x}), \widetilde{m}(\widetilde{x}), \nabla \widetilde{m}(\widetilde{x}) \nabla \psi\left(\psi^{-1}(\widetilde{x})\right)+\nabla \chi(s)\right) d s .
\end{aligned}
$$

The definition of $W_{0}$ implies that

$$
\begin{aligned}
& Q_{T}^{2,3} W_{0}\left(\psi^{-1}(\widetilde{x}), \widetilde{m}(\widetilde{x}), \nabla \widetilde{m}(\widetilde{x}) \nabla \psi\left(\psi^{-1}(\widetilde{x})\right)\right)=\inf _{\chi \in W_{0}^{1, \infty}\left(O ; \widetilde{m}(\widetilde{x})^{\perp}\right)} \frac{1}{\operatorname{meas}(O)} \int_{O} \inf _{z \in \widetilde{m}(\widetilde{x})^{\perp}} W(\widetilde{m}(\widetilde{x}) \\
&\left.,\left(\nabla \widetilde{m}(\widetilde{x}) \nabla \psi\left(\psi^{-1}(\widetilde{x})\right)+\nabla \chi(s) \mid z\right) A_{0}\left(\psi^{-1}(\widetilde{x})\right)\right) d s .
\end{aligned}
$$


Moreover, we have

$$
\begin{gathered}
\left(\nabla \widetilde{m}(\widetilde{x}) \nabla \psi\left(\psi^{-1}(\widetilde{x})\right)+\nabla \chi(s) \mid z\right) A_{0}\left(\psi^{-1}(\widetilde{x})\right)=\left(\nabla \widetilde{m}(\widetilde{x}) \nabla \psi\left(\psi^{-1}(\widetilde{x})\right) \mid 0\right) A_{0}\left(\psi^{-1}(\widetilde{x})\right) \\
+(\nabla \chi(s) \mid 0) A_{0}\left(\psi^{-1}(\widetilde{x})\right)+(0 \mid z) A_{0}\left(\psi^{-1}(\widetilde{x})\right)
\end{gathered}
$$

We also have

$$
\begin{aligned}
\left(\nabla \widetilde{m}(\widetilde{x}) \nabla \psi\left(\psi^{-1}(\widetilde{x})\right) \mid 0\right) & =\nabla \widetilde{m}(\widetilde{x})\left(\nabla \psi\left(\psi^{-1}(\widetilde{x})\right) \mid 0\right) \\
& =\nabla \widetilde{m}(\widetilde{x})\left(a_{1}\left(\psi^{-1}(\widetilde{x})\right)\left|a_{2}\left(\psi^{-1}(\widetilde{x})\right)\right| 0\right) .
\end{aligned}
$$

The condition $m_{, 3}=0$ becomes $\nabla \widetilde{m}(\widetilde{x}) a_{3}\left(\psi^{-1}(\widetilde{x})\right)=0$ and so we have

$$
\nabla \widetilde{m}(\widetilde{x})\left(\nabla \psi\left(\psi^{-1}(\widetilde{x})\right) \mid 0\right)=\nabla \widetilde{m}(\widetilde{x})\left(a_{1}\left(\psi^{-1}(\widetilde{x})\right)\left|a_{2}\left(\psi^{-1}(\widetilde{x})\right)\right| a_{3}\left(\psi^{-1}(\widetilde{x})\right)\right) .
$$

We also have

$$
\begin{aligned}
A_{0}(x) & =\nabla \Psi^{-1}\left(\Psi\left(x_{1}, x_{2}, 0\right)\right) \\
& =\left(\nabla \Psi\left(x_{1}, x_{2}, 0\right)\right)^{-1} \\
& =\left(a_{1}\left(\Psi^{-1}(\widetilde{x})\right)\left|a_{2}\left(\psi^{-1}(\widetilde{x})\right)\right| a_{3}\left(\Psi^{-1}(\widetilde{x})\right)\right)^{-1},
\end{aligned}
$$

which implies that

$$
\nabla \widetilde{m}(\widetilde{x})\left(\nabla \psi\left(\psi^{-1}(\widetilde{x})\right) \mid 0\right) A_{0}\left(\psi^{-1}(\widetilde{x})\right)=\nabla \widetilde{m}(\widetilde{x}) .
$$

Next, we set $\bar{s}=\nabla \psi\left(\psi^{-1}(\widetilde{x})\right) s$ and $\bar{\chi}(\bar{s})=\chi(s)$. We have $\bar{\chi} \in W_{0}^{1, \infty}\left(O_{a_{3}\left(\psi^{-1}(\widetilde{x})\right)} ; \mathbb{R}^{3}\right)$ and

$$
(\nabla \chi(s) \mid 0) A_{0}\left(\psi^{-1}(\widetilde{x})\right)=D_{a_{3}\left(\psi^{-1}(\widetilde{x})\right)^{\perp}} \bar{\chi}(\bar{s}) .
$$

Finally, for every $z \in \mathbb{R}^{3}$ we have

$$
(0 \mid z) A_{0}\left(\psi^{-1}(\widetilde{x})\right)=z \otimes a_{3}\left(\psi^{-1}(\widetilde{x})\right) .
$$

Choosing $O=\left(\nabla \psi\left(\psi^{-1}(\widetilde{x})\right)\right)^{-1} O_{a_{3}\left(\psi^{-1}(\widetilde{x})\right)}$ and replacing (6.2), (6.3) and (6.4) in (6.1), we obtain

$$
\begin{aligned}
& Q_{T}^{2,3} W_{0}\left(\psi^{-1}(\widetilde{x}), \widetilde{m}(\widetilde{x}), \nabla \widetilde{m}(\widetilde{x}) \nabla \psi\left(\psi^{-1}(\widetilde{x})\right)\right) \\
& =\inf _{\chi \in W_{0}^{1, \infty}\left(O_{a_{3}\left(\psi^{-1}(\widetilde{x})\right)} ; \widetilde{m}^{\perp}\right)}\left[\frac { 1 } { \operatorname { m e a s } ( O _ { a _ { 3 } ( \psi ^ { - 1 } ( \widetilde { x } ) ) } ) } \int _ { O _ { a _ { 3 } ( \psi ^ { - 1 } ( \widetilde { x } ) ) } } \left\{\operatorname { i n f } _ { z \in \widetilde { m } ^ { \perp } } W \left(\widetilde{m}, F+z \otimes a_{3}\left(\psi^{-1}(\widetilde{x})\right)\right.\right.\right. \\
& \left.\left.\left.+D_{a_{3}\left(\psi^{-1}(\widetilde{x})\right)} \chi \chi(s)\right)\right\} d s\right],
\end{aligned}
$$

and thus the result. 


\subsection{Model with external magnetic field}

If the curved film is placed in a uniform external magnetic field $H_{e}$, the free energy governing the behavior of the film will contain a term of external energy $E_{\text {ext }}$, called the Zeeman energy, depending on the magnetization $\widetilde{m}_{h}$ of the film via

$$
\widetilde{E}_{\text {ext }}\left(\widetilde{m}_{h}\right)=\frac{1}{h} \int_{\widetilde{\Omega}_{h}}\left(H_{e}, \widetilde{m}_{h}\right) d \widetilde{x} .
$$

Setting for all $x \in \Omega_{1}, m(h)(x)=\widetilde{m}\left(\Psi\left(x_{1}, x_{2}, h x_{3}\right)\right)$ and $E_{\text {ext }}(h)(m(h)(x))=\widetilde{E}_{\text {ext }}\left(\widetilde{m}_{h}\right)$ we obtain

$$
E_{\text {ext }}(h)(m(h))=\int_{\Omega_{1}}\left(H_{e}, m(h)\right) d_{h} d x .
$$

When $m(h)$ converges to $m(0)$ strongly in $L^{2}\left(\Omega_{1} ; \mathbb{R}^{3}\right)$ with $m(0)_{, 3}=0$ in $\Omega_{1}$, we have for a subsequence still denoted $h$

$$
\begin{aligned}
E_{\text {ext }}(h)(m(h)) & \rightarrow E_{\text {ext }}(0)(m(0)) \\
& =\int_{\omega}\left(H_{e}, m(0)\right) d_{0} d x,
\end{aligned}
$$

and the $\Gamma$-convergence analysis remains unchanged. The total limit energy will be

$$
\begin{aligned}
e(0)(m(0))=\int_{\omega}\left\{Q_{T}^{2,3} W_{0}(x, m(0)(x),(m(0), 1 \mid m(0), 2))\right. & +\left|\left(a_{3}(x), m(0)\right)\right|^{2} \\
& \left.+\left(H_{e}, m(0)\right)\right\} d_{0} d x
\end{aligned}
$$

and its expression on the curved surface is

$$
\widetilde{e}(0)(\widetilde{m})=\int_{\widetilde{S}}\left\{\widetilde{W}(\widetilde{n}(\widetilde{x}), \widetilde{m}, \nabla \widetilde{m}(\widetilde{x}))+|(\widetilde{n}(\widetilde{x}), \widetilde{m}(\widetilde{x}))|^{2}+\left(\widetilde{H}_{e}, \widetilde{m}\right)\right\} d \widetilde{x} .
$$

\subsection{The curved Gioia and James model}

We apply our results to the particular case of the Gioia and James model [8], that is with $W(y, F)=\varphi(y)+\alpha|F|^{2}$. The limit energy reads

$$
\widetilde{e}(0)(\widetilde{m})=\int_{\widetilde{S}}\{\widetilde{W}(\widetilde{n}(\widetilde{x}), \widetilde{m}, \nabla \widetilde{m}(\widetilde{x}))+|(\widetilde{n}(\widetilde{x}), \widetilde{m}(\widetilde{x}))|\} d \widetilde{x},
$$

with

$$
\begin{aligned}
& \widetilde{W}(\widetilde{n}(\widetilde{x}), \widetilde{m}, \nabla \widetilde{m}(\widetilde{x})) \\
= & \inf _{\chi \in W_{0}^{1, \infty}\left(O_{\widetilde{n}} ; \widetilde{m}^{\perp}\right)}\left[\frac{1}{\operatorname{meas}\left(O_{\widetilde{n}}\right)} \int_{O_{\widetilde{n}}}\left\{\inf _{z \in \widetilde{m}^{\perp}}\left[\varphi(\widetilde{m})+\alpha\left|\nabla \widetilde{m}+z \otimes \widetilde{n}+D_{\widetilde{n}^{\perp}} \chi(s)\right|^{2}\right]\right\} d s\right] .
\end{aligned}
$$


We have that

$$
\begin{aligned}
\left|\nabla \widetilde{m}+z \otimes \widetilde{n}+D_{\widetilde{n}^{\perp}} \chi(s)\right|^{2} & =|\nabla \widetilde{m}|^{2}+|z \otimes \widetilde{n}|^{2}+\left|D_{\widetilde{n}^{\perp}} \chi(s)\right|^{2} \\
& +2 \nabla \widetilde{m}: z \otimes \widetilde{n}+2 \nabla \widetilde{m}: D_{\widetilde{n} \perp} \chi(s)+2 z \otimes \widetilde{n}: D_{\widetilde{n}^{\perp}} \chi(s) .
\end{aligned}
$$

Since $\nabla \widetilde{m} \widetilde{n}=0$, this implies that $\nabla \widetilde{m}: z \otimes \widetilde{n}=0$. We also have, since $D_{\widetilde{n} \perp} \chi(s) \widetilde{n}=0$, that $z \otimes \widetilde{n}: D_{\widetilde{n}^{\perp}} \chi(s)=0$. Thus, we have

$$
\left|\nabla \widetilde{m}+z \otimes \widetilde{n}+D_{\widetilde{n} \perp} \chi(s)\right|^{2}=|\nabla \widetilde{m}|^{2}+|z \otimes \widetilde{n}|^{2}+\left|D_{\widetilde{n} \perp} \chi(s)\right|^{2}+2 \nabla \widetilde{m}: D_{\widetilde{n} \perp} \chi(s) .
$$

This means that the infimum for $z \in \widetilde{m}^{\perp}$ is reached when $z$ belongs to $\widetilde{n}^{\perp} \cap \widetilde{m}^{\perp}$ in particular for $z=0$. Thus, we obtain

$$
\begin{aligned}
& \widetilde{W}(\widetilde{n}(\widetilde{x}), \widetilde{m}, \nabla \widetilde{m}(\widetilde{x}))=\inf _{\chi \in W_{0}^{1, \infty}\left(O_{\tilde{n}} ; \widetilde{m}^{\perp}\right)}\left[\frac{1}{\operatorname{meas}\left(O_{\widetilde{n}}\right)} \int_{O_{\widetilde{n}}}\left[\varphi(\widetilde{m})+\alpha\left|\nabla \widetilde{m}+D_{\widetilde{n}^{\perp}} \chi(s)\right|^{2}\right] d s\right] \\
& =\inf _{\chi \in W_{0}^{1, \infty}\left(O ; \widetilde{m}^{\perp}\right)}\left[\frac{1}{\operatorname{meas}(O)} \int_{O}\left[\varphi(\widetilde{m})+\alpha\left|\nabla \widetilde{m}+(\nabla \chi(s) \mid 0) A_{0}\left(\psi^{-1}(\widetilde{x})\right)\right|^{2}\right] d s\right],
\end{aligned}
$$

with $O$ an arbitrary bounded open domain of $\mathbb{R}^{2}$. The invertibility of the matrix $A_{0}\left(\psi^{-1}(\widetilde{x})\right)$ implies that

$$
\begin{aligned}
\widetilde{W}(\widetilde{n}(\widetilde{x}), \widetilde{m}, \nabla \widetilde{m}(\widetilde{x})) & =\inf _{\chi \in W_{0}^{1, \infty}\left(D ; \widetilde{m}^{\perp}\right)}\left[\frac{1}{\operatorname{meas}(D)} \int_{D}\left[\varphi(\widetilde{m})+\alpha|\nabla \widetilde{m}+\nabla \chi(s)|^{2}\right] d s\right] \\
& =\varphi(\widetilde{m})+\alpha Q_{T}^{3,3} \Phi(\nabla \widetilde{m}),
\end{aligned}
$$

with $\Phi: M^{3} \rightarrow \mathbb{R}$ defined by $\Phi(A)=|M|^{2}$ and $D$ an arbitrary bounded open domain of $\mathbb{R}^{3}$. Since $\Phi$ is convex, its tangential quasiconvex envelope is $\Phi$ itself and we have $\widetilde{W}(\widetilde{n}(\widetilde{x}), \widetilde{m}, \nabla \widetilde{m}(\widetilde{x}))=\varphi(\widetilde{m})+\alpha|(\nabla \widetilde{m})|^{2}$, therefore

$$
\widetilde{e}(0)(\widetilde{m})=\int_{\widetilde{S}}\left\{\varphi(\widetilde{m})+\alpha|(\nabla \widetilde{m})|^{2}+\left|\left(\widetilde{n}\left(\psi^{-1}(\widetilde{x})\right), \widetilde{m}(\widetilde{x})\right)\right|^{2}\right\} d \widetilde{x}
$$

And thus we have a generalization of the results of [8].

Acknowledgements. I wish to thank Professor Hervé Le Dret for many useful discussions concerning the subject of this paper.

\section{References}

[1] E. Acerbi, G. Buttazzo and D. Percivale, A variational definition for the strain energy of an elastic string. Journal of Elasticity 25, (1991), 137-148. 
[2] R. AliCANDRo and C. LEONE, 3D-2D Asymptotic analysis for micromagnetic thin films, ESAIM: COCV, 6 (2001), 489-498.

[3] W.F. Brown, Micromagnetics, New York: Wiley, 1963.

[4] B. DaCorogna and I. FonseCA, Minima absolus pour des énergies ferromagnétiques. C. R. Acad. Sci. Paris, t. 331, Série 1, p. 497-500, 2000.

[5] B. Dacorogna, I. Fonseca, J. Malỳ and K. Trivisa, Manifold constrained variational problems. Calc. Var.9 (1999), 185-206.

[6] A. De Simone, Energy minimizers for large ferromagnetic bodies. Arch. Rational Mech. Anal. 125, (1993) 99-143.

[7] I. EKeland and R. TEMAM, Analyse convexe et problèmes variationnels. Dunod, Paris.

[8] G. Gioia and R.D. James, Micromagnetics of very thin films. Proc. Roy. Soc. Lond. Ser. A 453 (1997) 213-223.

[9] L.D. LANDAU and E.M. LIFSCHITZ, On the theory of the dispertion of magnetic permeability in ferromagnetic bodies. Phys. Z. Sowjetunion 8 (1935), 153-169.

[10] H. LE DRET and A. RAOULT, The nonlinear membrane model as variational limit of nonlinear three-dimensional elasticity. J. Math. Pures Appl. (9), 74(6): 549-578, 1995.

[11] H. LE DRET and A. RAOULT, The membrane shell model in nonlinear elasticity: A variational asymptotic derivation. J. Nonlinear Sci: 59-84, 1996.

[12] P. MARCELlini, Approximation of quasiconvex functions and lower semicontinuity of multiple integrals. Manuscripta Math. 51 (1985) 1-28

[13] H. Zorgati, Modélisation de films minces, Doctoral dissertation, Université de Pierre et Marie Curie, Paris, 2004.

[14] H. Zorgati, Films courbés minces ferromagnétiques. C. R. Acad. Sci. Paris, Ser. I 340 (2005) 81-86. 